\title{
Mapeamento sistemático da literatura para compras públicas sustentáveis
}

\section{Vanessa de Oliveira ${ }^{1}$ e Roberta Dalvo Pereira da Conceição $^{2}$}

${ }^{1}$ Instituto Federal do Rio de Janeiro. Reitoria. Rua Buenos Aires, 256. Centro. Rio de Janeiro-RJ, Brasil (CEP 20070-022). E-mail: livhug@hotmail.com.

${ }^{2}$ Centro Federal de Educação Tecnológica Celso Suckow da Fonseca. Campus Petrópolis. Rua do Imperador, 971. Centro. Petrópolis-RJ, Brasil (CEP 25620-971).

Resumo. Toda pesquisa tem seu início com a construção de uma base de conhecimento a respeito de determinado assunto, para isso, o pesquisador realiza uma análise de publicações científicas e literárias, que embasam seu estudo. 0 Mapeamento Sistemático da Literatura é um estudo secundário, que foi utilizado para esse trabalho com o intuito de buscar uma literatura que identificasse pesquisas relacionadas a abordagens de apoio para a rastreabilidade de temas que envolvam, de uma forma geral, compras públicas sustentáveis e responder questões sobre tendências de pesquisas na área. Sendo assim, objetivo deste artigo é relatar os resultados do estudo realizado. 0 intuito era criar uma base inicial para fundamentar a pesquisa com tema específico sobre compras públicas sustentáveis, assim como, elaborar um catálogo de pesquisas que poderão servir de base para futuras pesquisas sobre algum tema dentro do assunto relacionado ao desenvolvimento sustentável. Para uma utilização correta do mapeamento sistemático (MS), foi necessário o desenvolvimento de três etapas que são: planejamento da revisão, condução da revisão e o relatório da revisão. As publicações foram analisadas e as que se destacavam em relação aos assuntos referentes ao desenvolvimento sustentável e gestão sustentável, de maneira a contribuir, assim como relacionados a compras ou contratações sustentáveis, compras e contratações públicas sustentáveis e que de alguma forma geravam um desafio para o estudo, foram selecionados. Foram apresentados os resultados, onde o processo de busca retornou 6.763 estudos, dos quais 306 estudos relevantes inicialmente foram selecionados. Esses estudos, após a extração, diminuíram para 111 estudos classificadas como relevantes e de qualidade, que formaram uma base inicial para a pesquisa. Os estudos selecionados apresentaram alguns desafios e apresentaram investigações sobre economia circular e gerenciamento de cadeia de suprimentos verdes, os quais apresentaram imaturidade em suas áreas de pesquisa e podem ser temas para futuros trabalhos.
Recebido

$12 / 07 / 2020$

Aceito

$10 / 11 / 2020$

Disponível on line

$11 / 11 / 2020$

Publicado

$31 / 12 / 2020$

Acesso aberto

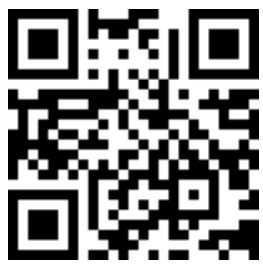

ORCID

(1) 0000-0002-8620-8659

Vanessa de Oliveira

D 0000-0002-1566-0437 Roberta Dalvo Pereira da Conceição

ISSN 2359-1412/RBGAS-2020-0106/2020/7/17/23/1391

Rev. Bras. Gest. Amb. Sustent.

http://revista.ecogestaobrasil.net 
Palavras-chave: Mapeamento sistemático; Compras sustentáveis; Desenvolvimento Sustentável.

Abstract. Systematic literature mapping for a study on sustainable public purchases. Every research begins upon the construction of a knowledge base regarding such matter, thereunto the researcher analyses scientific and literary publications that base his study. The Systematic Mapping of the Literature is a secondary study that was used in this work in order to search such a literature that could identify researches related to supportive approaches for the traceability of topics involving, in general, sustainable public procurement and answer questions on research trends in the area. Thus, this article aims at reporting the results of the performed study. The goal was to create an initial base to ground researches regarding specifically sustainable public procurement, as well as elaborating a research catalog that might ground future researches about any theme regarding sustainable development. For the adequate usage of the Systematic Mapping (SM) it was necessary the development of three stages: review planning, review performing, and review reporting. The publications were analyzed and the ones that stood out relating to subjects on sustainable development and management in order to contribute as well as related to sustainable procurement or contracting, sustainable public procurement and contracting and that somehow create a challenge for the study were selected. The results were presented where the research process retrieved 6.763 studies, of which 306 relevant studies were initially selected. Those studies, after the extraction, decreased to 111 relevant and quality studies that built an initial base for the research. The selected studies presented some challenges and inquiries about circular economy and green supply chain management that showed immaturity in its research areas and might be a theme for future research.

Keywords: Systematic mapping; Sustainable purchasing; Sustainable development.

\section{Introdução}

Toda pesquisa tem seu início com a construção de uma base de conhecimento a respeito de determinado assunto, para isso, o pesquisador realiza uma análise de publicações científicas e literárias, que embasam seu estudo. Essa análise é realizada através de uma revisão da literatura ou um mapeamento sistemático. Para esse estudo foi utilizado o método do mapeamento, que serviu para estruturar a pesquisa desenvolvida para a busca por estudos relacionados a compras públicas sustentáveis, onde 0 pesquisador pode avaliar a relevância ou não do material coletado e assim, construir sua própria base científica para fundamentar seus argumentos levantados. Para Gil (2008, p.60) "qualquer que seja a pesquisa, a necessidade de consultar material publicado é imperativa". Complementa ainda que, a revisão bibliográfica em publicações relevantes pode "identificar o estágio em que se encontram os conhecimentos acerca do tema que está sendo investigado" (Gil, 2008, p. 60). 
O mapeamento sistemático da literatura é um estudo secundário, que foi utilizado para esse trabalho com o intuito de buscar uma literatura que identificasse pesquisas relacionadas a abordagens de apoio para a rastreabilidade de temas que envolvam, de uma forma geral, compras públicas sustentáveis e responder questões sobre tendências de pesquisas na área.

Além disso, o propósito desse estudo foi realizar uma busca em fontes confiáveis, as quais tivessem como foco, descobrir quais as abordagens mais atuais descritas na literatura existente para auxiliar a rastreabilidade de requisitos, que poderiam ser de grande valia para o objetivo final da pesquisa de mestrado desenvolvida, que era propor critérios para a implantação de práticas de gestão sustentável nas licitações do Instituto Federal do Rio de Janeiro (IFRJ). Com base no seu planejamento anual de compras. Cabe destacar que as fontes de busca foram buscadas através da plataforma do Portal de Periódicos, da Fundação Coordenação de Aperfeiçoamento de Pessoal de Nível Superior (CAPES).

Sendo assim, objetivo deste artigo é relatar os resultados do estudo realizado, que teve seu foco na elaboração de um levantamento através do Mapeamento Sistemático de Literatura. Para começar, o estudo selecionou uma ampla área que envolveu assuntos relacionados ao desenvolvimento sustentável, gestão sustentável e depois restringiu a compras sustentáveis e compras públicas sustentáveis, critérios e indicadores sustentáveis. 0 intuito era criar uma base inicial para fundamentar a pesquisa com tema específico sobre compras públicas sustentáveis, assim como, elaborar um catálogo de pesquisas que poderão servir de base para futuras pesquisas sobre algum tema dentro do assunto relacionado ao desenvolvimento sustentável.

Os estudos foram coletados de vários periódicos internacionais disponíveis, como Scopus, Taylor e Francis Online e Science Direct. Mais de 6.000 publicações foram reunidas, no entanto, apenas 111 artigos foram escolhidos devido à alta coesão com o objetivo deste artigo. Todos eles eram analisados de forma independente e depois classificados por questões discutidas e pelo modelo utilizado na pesquisa, conforme apresentado no protocolo da revisão.

\section{Desenvolvimento sustentável e as compras públicas sustentáveis}

Desenvolvimento sustentável não é um assunto tão novo mais para a maioria das pessoas e instituições. Muitas instituições privadas têm adotado políticas sustentáveis em suas estratégias de negócios. Já a administração pública por ser, de alguma forma, grande influenciadora para que transformações ocorram em toda a sociedade, tem a necessidade de se posicionar a respeito do tema e, assim, criar ações e políticas que incentivem cada vez mais o desenvolvimento sustentável no país.

Sobre políticas públicas voltadas para atender o desenvolvimento sustentável num país através das compras públicas sustentáveis, Foukaras e Toma (2014) acreditam que direcioná-las para determinados fatores, como serviços de reciclagem, mudanças de legislações, melhoria no acesso à informação sobre os impactos ambientais a diversos grupos da população, criação de uma política de rotulagem de produtos ecológicos, todos esses fatores estão relacionadas questões econômicas, institucionais, organizacionais e culturais de um país.

Porém, cabe ressaltar que desenvolvimento sustentável é um assunto bastante amplo e envolve diversos temas. Diante disso, a pesquisa era sobre compras públicas sustentáveis, ao qual o estudo será limitado.

Para Aldenius e Kahn (2017, p. 251) “as compras públicas sustentáveis podem ter um objetivo mais amplo e incluir todos os pilares da sustentabilidade - econômico, social e ambiental". Ou seja, todo agente público pode e deve prever em seus planejamentos de compras os três pilares da sustentabilidade, buscando, assim, adquirir e contratar atendendo a todos os seus critérios. Para Biderman et al. (2008) compras sustentáveis é a

Rev. Bras. Gest. Amb. Sustent., 2020, vol. 7, n. 17, p. 1391-1423. 
maneira com que os profissionais de compras, podem integrar temas que dizem respeito ao ambiente, as questões sociais e, de certa forma, prever questões econômicas, todos os três pilares avaliados em todas as fases do processo da aquisição de bens ou contratação de serviços, buscando focar na redução dos impactos à saúde humana, ao meio ambiente e aos direitos humanos, com intuito de atender as necessidades dos consumidores finais.

Vale destacar que a Organização das Nações Unidas (ONU, 2018) incluiu na Agenda 2030 para o Desenvolvimento Sustentável, um objetivo que inclui ações que dizem respeito a compras sustentáveis, trata-se do Objetivo 12, sobre a produção e o consumo sustentáveis, que apresenta a ação "promover práticas de compras públicas sustentáveis, de acordo com as políticas e prioridades nacionais, onde todos os países deverão se envolver e tomar as medidas necessárias" (ONU, 2018). Ou seja, os governantes dos países devem promover o desenvolvimento sustentável, também por meio das compras públicas sustentáveis.

Para Buniamin et al. (2016), com o aumento das questões ambientais, as práticas de compras públicas sustentáveis, de forma eficaz, está sendo cada vez mais exigida. Para esses autores "tais práticas podem ajudar a promover a meta de desenvolvimento sustentável e abordar questões ambientais através de compras e facilitando o uso de produtos e serviços ambientalmente amigáveis" (Buniamin et al., 2016, p. 33)

O ICLEI (2019), uma rede global comprometida com o desenvolvimento sustentável em todos os países, criou o programa Rede Global de Cidades Líderes em Compras Sustentáveis (Global Lead City Network on Sustainable Procurement), que atualmente conta com 14 cidades, que se comprometeram a conduzir uma transição do estado atual de consumo e produção para estes de forma mais sustentável, através da implementação de compras sustentáveis e inovadoras. 0 intuito deste programa é levar à sociedade o uso de recursos eficientes, com baixa emissão de carbono e socialmente responsável.

Dentre estas 14 cidades, nove delas apresentam semelhanças com as ações já implantadas no Brasil e outras que podem servir como boas práticas para implementação ou melhorar alguma já existente. Quanto às semelhanças, tem-se, por exemplo, as compras compartilhadas, aquisição de equipamentos que economizam água e energia, incentivo às micro e às pequenas empresas, entre outros.

Para buscar mais informações a respeito de compras públicas sustentáveis, através de publicações científicas já divulgadas, realizou-se uma seleção de artigos publicados através do método de mapeamento sistemático da literatura, que é considerado um estudo secundário, e que contribuiu para a construção do conhecimento na pesquisa que foi desenvolvida.

\section{Metodologia}

Para esse estudo foi utilizado o método de mapeamento sistemático da literatura, que é um tipo de estudo secundário que realiza uma ampla revisão de estudos primários de um tema, que podem identificar evidências sobre este, como também podem identificar lacunas sobre possíveis novos estudos primários, que poderão ser necessários, além de poder identificar agrupamentos de evidências que podem levar a novas revisões sistemáticas (Kitchenham e Charters, 2007; Petersen et al., 2008; Kitchenham et al., 2010).

Por outro lado caso, caso fosse percebido, durante a execução do mapeamento sistemático, que se encontrou poucas publicações relevantes, com poucas evidências ou que o assunto é muito amplo, seria utilizado para contemplar a pesquisa bibliográfica a revisão sistemática da literatura, que também é um tipo de estudo secundário (Kitchenham e Charters, 2007).

Uma das justificativas para a realização desse tipo de pesquisa bibliográfica, através de revisão sistemático da literatura, está no fato de que se pode evidenciar um 
tratamento ou tecnologia, que pode resumir a evidência empírica de benefícios e até limitações de um método ágil específico (Kitchenham e Charters, 2007, p. 3). Além disso, Kitchenham e Charters (2007) descrevem como uma das vantagens desse método, que diz respeito quanto a relação do tipo de informações que são fornecidas sobre os efeitos de algum fenômeno ou até de métodos empíricos, se evidenciar que um fenômeno é robusto e transferível, os estudos possuem resultados consistentes, já se forem inconsistentes, as fontes é que poderão ser estudadas.

Este estudo contribuiu para fundamentar e embasar os argumentos ao questionamento da pesquisa, que estava sendo desenvolvida.

\section{Mapeamento sistemático da literatura}

Para Kitchenhm e Charters (2007) a revisão sistemática é um estudo que tem o intuito de identificar, avaliar e interpretar diversas pesquisas relevantes disponíveis e a quantidade de evidências encontradas de forma resumida, que podem auxiliar uma pesquisa individual. Parra tanto, como contribuição, foi realizado anterior à revisão, o mapeamento sistemático. Este, como já descrito por Kitchenham et al. (2009) é um estudo que contribui para que o pesquisador tenha um ponto de partida e outras ideias de pesquisa. Ainda destacam que os estudos de mapeamento podem ajudar as revisões sistemáticas nas áreas em que há uma grande quantidade de pesquisas, detalhando-as, principalmente onde há pouca pesquisa e com isso exigem mais pesquisa teórica e empírica (Kitchenham et al., 2009, p. 12).

Petersen et al. (2008) acredita que o estudo feito através do mapeamento sistemático é estruturado por fornecer publicações por meio de relatórios e resultados de pesquisa, onde podem ser categorizados e ter um mapa de seus resultados vistos num resumo visual. Essa visão geralmente não requer tantos esforços e, geralmente, requer menos esforços e fornece uma visão geral mais pontual.

Além disso, quando da análise de resultados, permite verificar com que frequência são publicadas por categorias e, dessa forma, visualizar a ênfase dada em pesquisas anteriores e, assim, identificar lacunas numa pesquisa atual, com o intuito de sugerir ou instigar novas hipóteses, novos estudos, um estudo adicional e contextualizar novas atividades de pesquisa (Petersen et al., 2008).

Para uma utilização correta, tanto do mapeamento sistemático (MS) quanto da revisão sistemática de literatura (RSL), foi necessário o desenvolvimento de três etapas que são: planejamento da revisão, condução da revisão e o relatório da revisão.

No planejamento, o objetivo foi identificar a necessidade de se realizar um mapeamento sistemático e revisão sistemática de literatura, nesse sentido, primeiramente, definiu-se o protocolo da revisão, que especificou as questões de pesquisas abordadas e direcionou-as para os métodos que foram utilizados para realizar a revisão (Kitchenham e Charters, 2007).

Concluída a primeira etapa, iniciou-se a etapa de condução da revisão. Através da definição do protocolo, selecionou-se os estudos através de alguns critérios, como: a busca e seleção de inclusão e exclusão (Kitchenham e Charters, 2007).

A terceira etapa foi a publicação dos resultados, através de um registro de todas as informações extraídas, que puderam ser também através de dissertações, teses, artigos científicos, através de tabelas, planilhas, relatórios técnicos.

Importante destacar, que apesar do mapeamento sistemático e revisão sistemática terem semelhanças e apoiarem a tomada de decisão relacionada a uma pesquisa em desenvolvimento entre eles (Falbo et al., 2017, p. 1), Petersen et al. (2015, p. 2) descreve algumas das diferenças que existem entre elas, como as relacionadas às questões de pesquisa, os critérios utilizados como estratégias de pesquisa, a avaliação da qualidade e resultados obtidos. As questões de pesquisa nos estudos de mapeamento são mais amplas e em maior número, pois tendem a descobrir tendências de pesquisa, são mais 
abrangentes e não se faz necessário a realização de uma avaliação de qualidade. Já as revisões sistemáticas têm o intuito de agregar evidências, são mais focadas, requer tempo e essencial que se realize uma avaliação de qualidade (Petersen et al., 2015, p. 2).

Reforçando as diferenças existentes entre o mapeamento sistemático e revisão sistemática, Kitchenhm e Charters (2007, p. 44) descreveram que "os resultados de um estudo de mapeamento podem identificar áreas adequadas para a realização de Revisões Sistemáticas da Literatura e áreas em que um estudo primário é mais apropriado" (tradução nossa). Sendo assim, elaborou-se a Tabela 1 para demonstrar as diferenças entre mapeamento sistemático e revisão sistemática, de acordo com Kitchenhm e Charters (2007, p. 44).

Tabela 1. Comparativo entre mapeamento sistemático (MS) e revisão sistemática de literatura (RSL).

\begin{tabular}{|l|l|l|}
\hline Característica & \multicolumn{1}{|c|}{ Mapeamento } & \multicolumn{1}{c|}{ Revisão } \\
\hline Questões de pesquisa & Mais amplas em maior número. & Mais focadas e em menor número. \\
\hline Termos usados & Mais abrangentes. & Altamente focados. \\
\hline Artigos retornados & Muitos. & Menos. \\
\hline Extração de Dados & $\begin{array}{l}\text { Mais ampla, com foco em } \\
\text { classificação, sem despender } \\
\text { muito tempo. }\end{array}$ & Precisa. Requer tempo \\
\hline Análise dos dados & $\begin{array}{l}\text { Basicamente faz uma } \\
\text { sumarização. Uso de gráficos. }\end{array}$ & $\begin{array}{l}\text { Profunda. Requer análise cuidadosa e } \\
\text { interpretação. }\end{array}$ \\
\hline $\begin{array}{l}\text { Disseminação de de limitada. Visa a influenciar a } \\
\text { resultados }\end{array}$ & $\begin{array}{l}\text { Mais limitacidar abrangente. Visa a elucidar } \\
\text { direção futura de estudos } \\
\text { primários. }\end{array}$ & $\begin{array}{l}\text { Mavos aspectos para investigação e } \\
\text { gerar evidências }\end{array}$ \\
\hline
\end{tabular}

Fonte: Adaptado de Kitchenham e Charters (2007, p. 44).

O estudo de mapeamento sistemático da literatura teve o apoio do software Start Lapes, elaborado pelo Laboratório de Pesquisa em Engenharia de Software da Universidade Federal de São Carlos (UFSCar).

Ao final, a pesquisadora tinha a disposição um catálogo de publicações relevantes ao seu tema de investigação, onde na sequência, foi necessário, realizar uma revisão sistemática da literatura.

\section{Protocolo da revisão}

Inicialmente, o mapeamento sistemático da literatura, iniciou seu estudo com a realização de um levantamento de referências teóricas em artigos científicos, teses, dissertações, trabalhos publicados em revistas, congressos, simpósios etc. Dessa forma, com as bases científicas já analisadas e publicadas por meios escritos e eletrônicos, direcionadas aos termos desenvolvimento sustentável, gestão sustentável, compras sustentáveis e compras públicas sustentáveis e critérios e indicadores sustentáveis, formariam um catálogo de publicações para embasar a pesquisa com o tema sobre compras públicas sustentáveis.

Para dar início ao mapeamento sistemático da literatura, foi preciso realizar as três etapas que são o planejamento, a realização e o relatório (Kitchenham e Charters, 2007). 
Inicialmente, foi definido uma lista de verificação, adaptada de uma lista apresentada por Kitchenham e Charters (2007) que incluiu o seguinte:

- Objetivo da revisão;

- Definição das questões a serem utilizadas na pesquisa a ser realizada;

- Definição dos locais e das fontes a serem pesquisadas para identificar os estudos primários, verificar se haverá restrições;

- Quais serão as palavras-chaves a serem utilizadas para pesquisar nas fontes

- Definição da cadeia de busca, o que chamam de string;

- Qual será o período a ser pesquisado;

- Critérios de inclusão e exclusão e como estes serão aplicados;

- Critérios utilizados para avaliar a qualidade dos estudos primários;

- Como será a extração dos dados dos estudos primários;

- Quais evidências serão encontradas, que contribuirão para responder o problema da pesquisa;

- Quantidade de pesquisas sobre o assunto relacionado à sustentabilidade que foram realizadas no período estipulado para a devida pesquisa.

Definido o planejamento, foi elaborado cada item da lista de verificação de acordo com o objetivo da realização do mapeamento sistemático da literatura e assim iniciou-se a revisão.

O mapeamento sistemático de literatura foi realizado com a ajuda do software Start, do Laboratório de Pesquisa em Engenharia de Software (LaPES), da Universidade Federal de São Carlos (UFSCar). Através deste software, onde foi elaborado o protocolo, na fase de planejamento da pesquisa, se definiu como seriam a inclusão e exclusão dos artigos encontrados nas fontes escolhidas e como seria a análise inicial para selecionar os artigos que, de fato, contribuiriam com a pesquisa.

Ao final, realizou-se uma análise mais criteriosa dos artigos com evidências encontradas e então se confeccionou um relatório com os dados relacionados à lista de verificação, assim como, também, uma análise dos resultados encontrados e assim relatálos.

\section{Planejamento}

Objetivo do presente estudo teve a intenção de explorar publicações relevantes e reconhecidas, que envolvessem assuntos sobre desenvolvimento sustentável, gestão sustentável, compras sustentáveis e compras públicas sustentáveis, assim como, sobre critérios e indicadores sustentáveis. Temas que pudessem contribuir para a pesquisa de mestrado que foi desenvolvida, que tem como objetivo final propor critérios para a implantação de práticas de gestão sustentável nas licitações do Instituto Federal do Rio de Janeiro (IFRJ), com base no seu planejamento anual de compras.

Definiram-se as fontes de busca através da plataforma do Portal de Periódicos, da Fundação Coordenação de Aperfeiçoamento de Pessoal de Nível Superior (CAPES), que é uma fonte confiável e vinculada a diversas instituições de ensino superior, as fontes selecionadas foram Springer Link, Scopus, Taylor \& Francis Online, Web of Science e Science Direct. Essas fontes foram definidas através de pesquisa à plataforma da CAPES.

Após definida as fontes, iniciou-se a etapa de criação do protocolo da pesquisa, onde se definiu a estratégia, os critérios, esse protocolo serviu para selecionar as publicações que realmente seriam relevantes e de qualidade para a pesquisa que foi realizada, ou seja, "um protocolo pré-definido é necessário para reduzir a possibilidade de viés do pesquisador" (Kitchenham e Charters, 2007, p. 12). 
- Q1: Quantos artigos a respeito dos assuntos mencionados no objetivo, ou próximo, foram publicados por ano desde 2014 ?

- Q2: Quantos artigos foram selecionados por serem relevantes quanto aos assuntos tratados?

- Q3: Quais foram as evidências encontradas nos artigos selecionados?

Em seguida, definido as fontes, estipulou-se as características de inclusão e exclusão. Sendo assim, uma das análises concentrou-se predominantemente em alguns critérios de inclusão (CI) que foram:

CI.1 - Publicações em inglês, espanhol e português.

CI.2 - Publicações abertas.

CI.3 - Artigos que falem de algum dos temas relacionados nas palavras chaves que tenham relação com a Agenda 2030 da ONU.

CI.4 - Artigos que falem sobre ciclo de vida, logística reversa, os 3R's (Reciclar, reutilizar e reduzir), porém que contribuam com os temas das palavras chaves.

CI.5 - Artigos com assuntos próximos e que tenham coerência com os assuntos das palavras chaves definidas.

Os itens de exclusões foram definidos de acordo com assuntos que não seriam relevantes ou tinham outro direcionamento, que o distanciava do objetivo desse estudo. Como itens de critério de exclusão (CE) foram estipulados os seguintes:

CE.1 - Artigos duplicados.

CE.2 - Artigos de idiomas diferentes do inglês, espanhol ou português.

CE.3 - Não tem acesso completo ao artigo (full paper).

CE.4 - Artigo não é relevante por não abordar sobre os temas informados como palavras chaves.

CE.5 - Artigos que falem sobre temas muito adversos ou que não contribuirão de alguma forma com o tema compras sustentáveis.

CE.6 - Artigos sem Abstract.

CE.7 - Artigos sem palavras-chaves.

Finalizada essa etapa, iniciou-se a condução de revisão da pesquisa por artigos que respondessem as questões e atendessem o objetivo desse estudo.

\section{Estratégia de busca}

Após a definição e como seria o processo de busca pelas fontes através das bibliotecas digitais, selecionadas na etapa do planejamento, iniciou-se a procura pelos estudos primários nas fontes selecionadas, que foram:

- Elsevier Science Direct (http://www.sciencedirect.com).

- Elsevier Scopus (http://www.scopus.com/home.url).

- $\quad$ Springer Link (http://link.springer.com).

- Taylor \& Francis (https://taylorandfrancis.com).

- Web of Science (https://webofknowledge.com).

Sendo que desses cinco, somente encontrou-se artigos relativos ao tema da pesquisa em Science Direct, Scopus e Taylor \& Francis. Nos demais não foram encontrados artigos primários disponíveis no modo aberto, por isso, foram excluídos do estudo.

Com as fontes selecionadas, definiu-se a string de busca de acordo com cada base escolhida. Foram selecionadas publicações entre os períodos de 2014 a 2019, estes estudos foram extraídos de três fontes de busca, dentre as cinco selecionadas, pois eram as 
que possuíam publicações que correspondiam ao objetivo do estudo. A Tabela 2 apresenta o string utilizado para cada uma destas fontes.

Tabela 2. Strings de busca e seus resultados numéricos.

\begin{tabular}{|c|c|c|}
\hline Fonte & Quantidade & String \\
\hline Science Direct & 5430 & $\begin{array}{lllll}\text { (sustainable AND development) AND } & \text { (sustainable } & \text { AND } \\
\text { purchasing) AND (sustainable AND management) } & \text { AND } \\
\text { (sustainable AND criteria OR indicators) } & & \\
\end{array}$ \\
\hline Scopus & 644 & $\begin{array}{l}\text { (sustainable AND development) AND (sustainable AND } \\
\text { purchasing) AND (sustainable AND public AND purchasing) } \\
\text { AND (sustainable AND management) AND (sustainable AND } \\
\text { criteria) AND (sustainable AND indicators) AND (LIMIT-TO } \\
\text { (PUBYEAR, 2020) OR LIMIT-TO (PUBYEAR, 2019) OR LIMIT- } \\
\text { TO (PUBYEAR, 2018) OR LIMIT-TO (PUBYEAR, 2017) OR } \\
\text { LIMIT-TO (PUBYEAR, 2016) OR LIMIT-TO (PUBYEAR, 2015) } \\
\text { OR LIMIT-TO (PUBYEAR, 2014)) AND (EXCLUDE (SUBJAREA, } \\
\text { "BIOC") OR EXCLUDE (SUBJAREA, "PSYC") OR EXCLUDE } \\
\text { (SUBJAREA, "CENG") OR EXCLUDE (SUBJAREA, "CHEM") OR } \\
\text { EXCLUDE (SUBJAREA, "NURS") OR EXCLUDE (SUBJAREA, } \\
\text { "PHAR") OR EXCLUDE (SUBJAREA, "PHYS") OR EXCLUDE } \\
\text { (SUBJAREA, "HEAL")) AND (EXCLUDE (SUBJAREA, "MEDI")) }\end{array}$ \\
\hline $\begin{array}{l}\text { Tylor \& Francis } \\
\text { online }\end{array}$ & 689 & $\begin{array}{l}\text { [All: sustainable development] AND [All: sustainable } \\
\text { purchasing] AND [All: sustainable public purchasing] AND [All: } \\
\text { sustainable criteria] AND [All: sustainable management] AND } \\
\text { [All: sustainable indicators] AND [Publication Date: } \\
(01 / 01 / 2014 \text { T0 } 12 / 31 / 2020)]\end{array}$ \\
\hline
\end{tabular}

Após um teste piloto para verificação de viabilidade de execução, chegou-se no formato, que melhor atendeu a necessidade, de acordo com cada fonte de busca. As palavras utilizadas foram todas na língua inglesa para facilitar a busca, pois houve um retorno maior.

\section{Condução da revisão}

A análise dos artigos selecionados se deu através da análise do título, palavraschaves e resumo. Desses, os estudos que não apresentavam desafios, relação com o tema da pesquisa, boas práticas, estudos de casos de sucesso, que não apresentavam proximidade nenhuma com qualquer uma das palavras chaves da pesquisa, eram claramente irrelevantes e que não atendiam a nenhum dos critérios de inclusão, foram excluídos nessa primeira análise.

A seleção de busca dessa metodologia utilizada para fundamentar a pesquisa em questão, não se limitou ao tipo de publicação, foram incluídos estudos que faziam parte de jornais, revistas, conferências, congressos, simpósios, relatórios técnicos e não descartou a possibilidade de pesquisas em teses, dissertações e livros.

Além disso, quando houve necessidade, foi realizado uma busca através de referenciais utilizados nos estudos primários encontrados.

\section{Avaliação da qualidade}

Kitchenham e Charters (2007) e Petersen et al. (2008; 2015), relatam a importância de se avaliar a qualidade dos estudos primários tanto no mapeamento sistemático quanto na revisão sistemática, para este trabalho, foi realizado uma avaliação 
da qualidade dos estudos selecionados. Apesar de não se ter uma definição universal do que se classifica como qualidade nos estudos primários, se torna difícil caracterizar estes estudos, como também descrito por Kitchenham e Charters (2007, p. 21), é "uma dificuldade inicial é que não há definição acordada de estudo qualidade do estudo".

Sendo assim, os estudos foram caracterizados como sendo de qualidade, aqueles que atendiam os seguintes critérios:

- Artigos que mencionaram algum tipo de critério sustentável, que possa ser considerado inovador e um desafio.

- Desafios encontrados nos artigos que envolvem desenvolvimento sustentável e gestão sustentável no que diz respeito a compras sustentáveis e compras públicas sustentáveis.

- Possui em seu artigo algum assunto relacionado ao desenvolvimento sustentável ou gestão sustentável que pode contribuir para a pesquisa.

- Artigos voltados para indicadores de sustentabilidade e que podem contribuir para a pesquisa, indicando a criação de critérios sustentáveis.

Com essa avaliação foi possível extrair e sintetizar os estudos que realmente importariam para contribuir de alguma forma com a pesquisa. A partir dessa etapa, realizou-se, então, uma análise mais detalhada de cada estudo selecionado para apresentar suas fundamentações como embasamento aos dados da pesquisa.

\section{Resultados}

Os resultados, que foram surgindo vieram dos mais diversos temas, pois sustentabilidade é muito amplo e pode ser utilizado para diversas razões e áreas, por isso a necessidade de se colocar uma segunda palavra para tentar filtrar até aproximar-se no objetivo do estudo.

A Tabela 3 ilustra a divisão das publicações selecionadas, após passar pelos critérios de inclusão e exclusão definidas no protocolo, respondendo também ao Q1, artigos publicados por ano.

Tabela 3. Resultados das publicações anuais.

\begin{tabular}{|l|c|c|c|}
\hline & Aceitos & Rejeitados & Duplicados \\
\hline 2014 & 35 & 772 & 7 \\
\hline 2015 & 73 & 950 & 6 \\
\hline 2016 & 57 & 1036 & 4 \\
\hline 2017 & 46 & 1169 & 5 \\
\hline 2018 & 49 & 1122 & 1 \\
\hline 2019 & 46 & 1376 & 9 \\
\hline Sem ano definido & 0 & 0 & \\
\hline
\end{tabular}

0 resultado do estudo é apresentado na Tabela 3 e é perceptível que nos últimos anos aumentou o número de publicações com as palavras definidas para serem utilizadas na busca. A busca resultou em 6.763 artigos publicados no período entre 2014-2019. A partir dessa fase, iniciou-se as exclusões, algumas foram feitas ainda no site de busca e algumas foram removidas simultaneamente, essa ação foi necessária para que as publicações mais relevantes e que realmente atendem o objetivo desse estudo, começassem a ser selecionados. 
Os artigos duplicados foram automaticamente excluídos, os demais foram analisados um a um conforme critérios definidos no protocolo. Os que eram classificados nos critérios de exclusão, foram considerados rejeitados. Para essa etapa foram lidos os seus resumos, títulos e palavras-chaves, onde se filtrou os artigos que não contribuíram para a pesquisa que foi desenvolvida.

Após a realização dessas exclusões, restaram 306 artigos que, inicialmente, foram considerados relevantes.

Dessas publicações, foi realizado a extração, etapa em que se busca nas publicações a qualidade de cada um deles, conforme descrito no protocolo. De acordo com Kitchenham e Charters (2007, p. 29) "os formulários de extração de dados devem ser projetados para coletar todas as informações necessárias para abordar as questões de revisão e os critérios de qualidade do estudo".

Neste momento, analisou as publicações que se destacavam em relação aos assuntos referentes ao desenvolvimento e gestão sustentável, de maneira a contribuir, assim como relacionados a compras ou contratações sustentáveis, compras e contratações públicas sustentáveis e que de alguma forma geravam um desafio para o estudo, foram selecionados. Não foi realizada uma exclusão de artigos do tipo quantitativo ou qualitativo, pois dependendo do seu conteúdo, mesmo que sendo quantitativo, poderia de alguma forma contribuir para o estudo.

Além desses buscou-se verificar nas publicações a existência de pesquisas que envolvessem critérios e indicadores sustentáveis para servirem de base para o estudo e a criação de critérios direcionados para a gestão sustentável das compras.

Após essa análise restaram então 111 publicações, classificadas como relevantes e de qualidade para o estudo e que poderão contribuíram para a formação de uma base de pesquisa. Nestes as palavras mais encontradas em seus títulos podem ser visualizadas na nuvem da Figura 1.

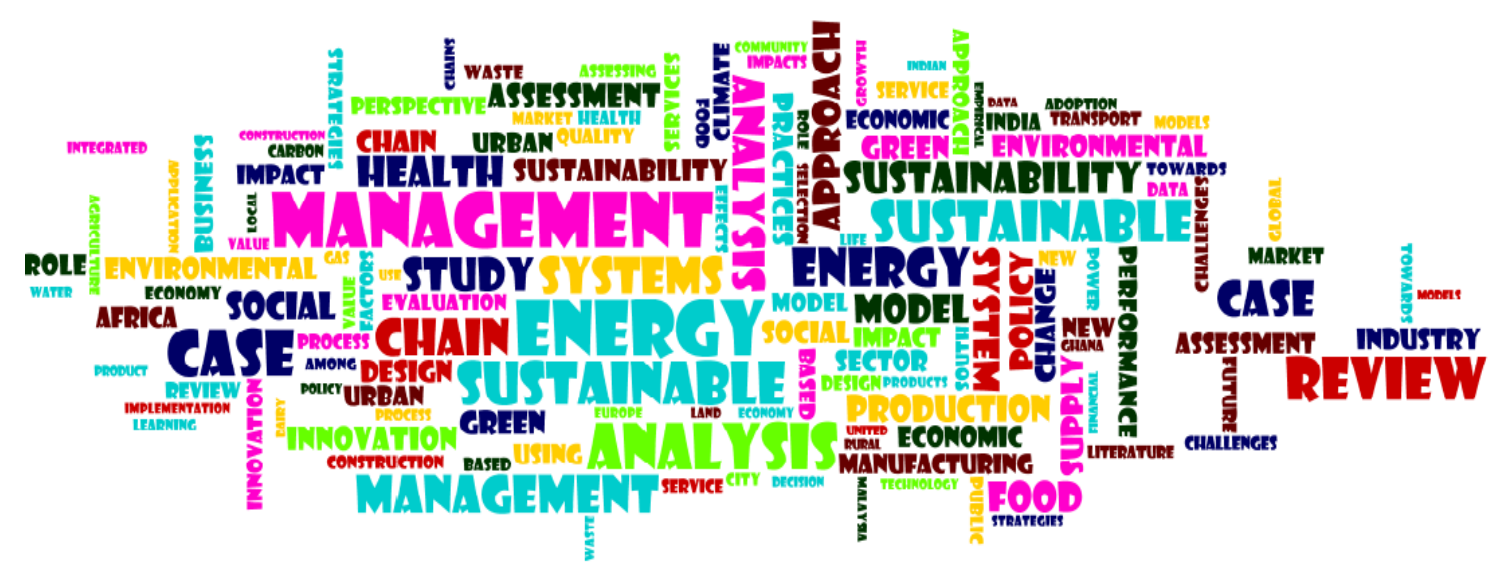

Figura 1. Nuvem de palavras mais citadas nos títulos das publicações selecionadas.

Algumas observações que podem ser visualizadas nessa nuvem. Muitas publicações voltadas para energia sustentável, gerenciamento da cadeia de suprimentos verdes e economia circular. Outra observação, que foi percebida além da nuvem e de uma forma geral pela autora, ao analisar os artigos, é que, aparentemente, a maioria das publicações é de países da Europa, como Reino Unido, Alemanha, Itália, Suécia, Romênia, 
etc., alguns países da África, como Gana, que está na nuvem inclusive, e diversos países da Ásia. Porém, desse último continente, o que chamou a atenção foi o grande número de publicações advindos da China e da Índia. Estes países estão investindo pesado em pesquisa na área de desenvolvimento, gestão e energia sustentável.

Em relação aos 111 artigos que foram selecionados, pode-se observar a quantidade de publicações por continente na Figura 2.

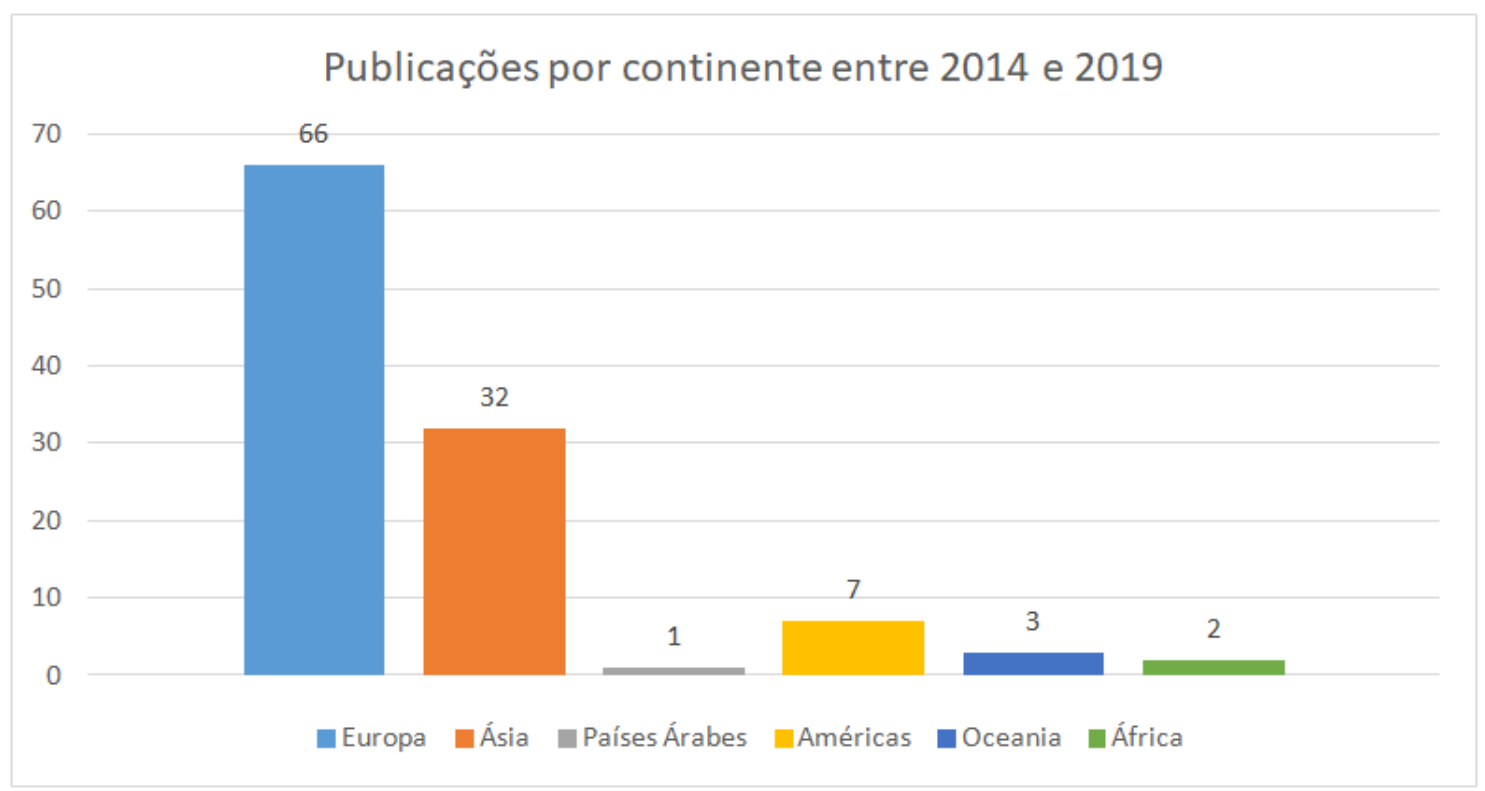

Figura 2. Publicações por continente entre 2014 a 2019.

Como pode ser observado, a Europa e a Ásia são os continentes que mais tem desenvolvido pesquisas na área de desenvolvimento sustentável. Cabe ressaltar que a Figura 2 foi elaborada com base nos 111 publicações selecionadas no estudo (Tabela 4).

Tabela 4. Publicações por continente entre 2014 a 2019.

\begin{tabular}{|l|l|c|l|c|}
\hline Continente & \multicolumn{1}{|c|}{ Autoria } & Ano & \multicolumn{1}{c|}{ Título } & Bases \\
\hline Africa & El Yamany et al. & 2016 & $\begin{array}{l}\text { Applicability and implementation } \\
\text { of U.S. green building council } \\
\text { rating system (LEED) in Egypt (A } \\
\text { longitudinal study for Egyptian } \\
\text { LEED Certified Buildings) }\end{array}$ & $\begin{array}{c}\text { Science } \\
\text { Direct }\end{array}$ \\
\hline Africa & Yadavalli et al. & \multirow{2}{*}{2019} & $\begin{array}{l}\text { An integrated optimization model } \\
\text { for selection of sustainable } \\
\text { suppliers based on customers } \\
\text { expectations }\end{array}$ & $\begin{array}{c}\text { Science } \\
\text { Direct }\end{array}$ \\
\hline
\end{tabular}


Tabela 4. Continuação.

\begin{tabular}{|c|c|c|c|c|}
\hline Continente & Autoria & Ano & Título & Bases \\
\hline América & $\begin{array}{l}\text { Chacón Vargas e Moreno } \\
\text { Mantilla }\end{array}$ & 2016 & $\begin{array}{l}\text { Organizational antecedents and } \\
\text { capabilities for sustainable supply } \\
\text { chain management in developing } \\
\text { economies: The case of Colombian } \\
\text { focal firms }\end{array}$ & Scopus \\
\hline América & Ingwersen et al. & 2016 & $\begin{array}{l}\text { Detailed life cycle assessment of } \\
\text { Bounty }{ }^{\circledR} \text { paper towel operations } \\
\text { in the United States }\end{array}$ & $\begin{array}{l}\text { Science } \\
\text { Direct }\end{array}$ \\
\hline América & Inti et al. & 2016 & $\begin{array}{l}\text { An approach for performing life } \\
\text { cycle impact assessment of } \\
\text { pavements for evaluating } \\
\text { alternative pavement designs }\end{array}$ & $\begin{array}{l}\text { Science } \\
\text { Direct }\end{array}$ \\
\hline América & Skerlos & 2015 & $\begin{array}{l}\text { Promoting effectiveness in } \\
\text { sustainable design }\end{array}$ & $\begin{array}{c}\text { Science } \\
\text { Direct }\end{array}$ \\
\hline América* & Costa e Motta & 2019 & $\begin{array}{l}\text { Key factors hindering sustainable } \\
\text { procurement in the Brazilian } \\
\text { public sector: A Delphi study }\end{array}$ & Scopus \\
\hline América* & Pereira e Silveira & 2014 & $\begin{array}{l}\text { A necessidade de adaptação às } \\
\text { regulações ambientais da política } \\
\text { nacional de resíduos sólidos: do } \\
\text { fabricante ao consumidor } \\
\text { organizacional no setor de } \\
\text { equipamentos eletromédicos }\end{array}$ & $\begin{array}{l}\text { Science } \\
\text { Direct }\end{array}$ \\
\hline América* & Pimenta e Ball & 2015 & \begin{tabular}{llr} 
Analysis of & \multicolumn{2}{c}{ environmental } \\
sustainability & practices & across \\
upstream & supply & chain \\
management & & \\
\end{tabular} & $\begin{array}{l}\text { Science } \\
\text { Direct }\end{array}$ \\
\hline Arábia & Mahdavinejad et al. & 2014 & $\begin{array}{l}\text { Dilemma of green and pseudo } \\
\text { green architecture based on LEED } \\
\text { norms in case of developing } \\
\text { countries }\end{array}$ & $\begin{array}{l}\text { Science } \\
\text { Direct }\end{array}$ \\
\hline Asia & Bae & 2017 & $\begin{array}{l}\text { The effect of environmental } \\
\text { capabilities on environmental } \\
\text { strategy and environmental } \\
\text { performance of Korean exporters } \\
\text { for green supply chain } \\
\text { management }\end{array}$ & $\begin{array}{l}\text { Science } \\
\text { Direct }\end{array}$ \\
\hline Asia & Bansia et al. & 2014 & $\begin{array}{l}\text { Development of a reverse logistics } \\
\text { performance measurement system } \\
\text { for a battery manufacturer }\end{array}$ & $\begin{array}{l}\text { Science } \\
\text { Direct }\end{array}$ \\
\hline Asia & Bidin et al. & 2018 & $\begin{array}{l}\text { Green related practices for } \\
\text { construction procurement }\end{array}$ & Scopus \\
\hline Asia & Buniamin et al. & 2016 & $\begin{array}{l}\text { Green Government Procurement } \\
\text { Practices (GGP) in Malaysian } \\
\text { public enterprises }\end{array}$ & $\begin{array}{l}\text { Science } \\
\text { Direct }\end{array}$ \\
\hline Asia & Chin et al. & 2015 & $\begin{array}{l}\text { Green supply chain management, } \\
\text { environmental collaboration and } \\
\text { sustainability performance }\end{array}$ & $\begin{array}{l}\text { Science } \\
\text { Direct }\end{array}$ \\
\hline
\end{tabular}


Tabela 4. Continuação.

\begin{tabular}{|c|c|c|c|c|}
\hline Continente & Autoria & Ano & Título & Bases \\
\hline Asia & Chun et al. & $2015 a$ & $\begin{array}{l}\text { Green supply chain management } \\
\text { in the construction industry: Case } \\
\text { of Korean construction companies }\end{array}$ & $\begin{array}{c}\text { Science } \\
\text { Direct }\end{array}$ \\
\hline Asia & Chun et al. & $2015 b$ & $\begin{array}{l}\text { Supply chain process and green } \\
\text { business activities: Application to } \\
\text { small and medium enterprises }\end{array}$ & $\begin{array}{l}\text { Science } \\
\text { Direct }\end{array}$ \\
\hline Asia & Diyar et al. & 2014 & $\begin{array}{l}\text { Green Economy: Innovation-based } \\
\text { development of Kazakhstan }\end{array}$ & $\begin{array}{c}\text { Science } \\
\text { Direct }\end{array}$ \\
\hline Asia & Eryigit e Ozcure & 2015 & $\begin{array}{l}\text { Eco-innovation as modern era } \\
\text { strategy of companies in } \\
\text { developing countries: Comparison } \\
\text { between Turkey and European } \\
\text { Union }\end{array}$ & $\begin{array}{c}\text { Science } \\
\text { Direct }\end{array}$ \\
\hline Asia & Galankashi et al. & 2015 & $\begin{array}{l}\text { Prioritizing green supplier } \\
\text { selection criteria using fuzzy } \\
\text { analytical network process }\end{array}$ & $\begin{array}{c}\text { Science } \\
\text { Direct }\end{array}$ \\
\hline Asia & Irfan et al. & 2018 & $\begin{array}{l}\text { Unravelling the fuzzy effect of } \\
\text { economic, social and } \\
\text { environmental sustainability on } \\
\text { the corporate reputation of public- } \\
\text { sector organizations: A case study } \\
\text { of Pakistan }\end{array}$ & Scopus \\
\hline Asia & Jayant e Azhar & 2014 & $\begin{array}{l}\text { Analysis of the barriers for } \\
\text { implementing Green Supply Chain } \\
\text { Management (GSCM) practices: An } \\
\text { Interpretive Structural Modeling } \\
\text { (ISM) Approach }\end{array}$ & $\begin{array}{c}\text { Science } \\
\text { Direct }\end{array}$ \\
\hline Asia & Jayant et al. & 2014 & $\begin{array}{l}\text { Simulation modelling and analysis } \\
\text { of network design for closed-loop } \\
\text { supply chain: A case study of } \\
\text { battery industry }\end{array}$ & $\begin{array}{c}\text { Science } \\
\text { Direct }\end{array}$ \\
\hline Asia & Jayanti e Gowda & 2014 & $\begin{array}{ll}\begin{array}{l}\text { Sustainability dilemmas in } \\
\text { emerging economies }\end{array} & \\
\end{array}$ & $\begin{array}{c}\text { Science } \\
\text { Direct }\end{array}$ \\
\hline Asia & Joshi e Rahman & 2015 & $\begin{array}{l}\text { Factors affecting green purchase } \\
\text { behaviour and future research } \\
\text { directions }\end{array}$ & $\begin{array}{c}\text { Science } \\
\text { Direct }\end{array}$ \\
\hline Asia & Karimi e Rahim & 2015 & $\begin{array}{l}\text { Classification of external } \\
\text { stakeholders pressures in green } \\
\text { supply chain management }\end{array}$ & $\begin{array}{c}\text { Science } \\
\text { Direct }\end{array}$ \\
\hline Asia & Kumar e Rahman & 2015 & $\begin{array}{l}\text { Sustainability adoption through } \\
\text { buyer supplier relationship across } \\
\text { supply chain: A literature review } \\
\text { and conceptual framework }\end{array}$ & $\begin{array}{c}\text { Science } \\
\text { Direct }\end{array}$ \\
\hline Asia & Ma et al. & 2019 & $\begin{array}{l}\text { Research on the complexity of } \\
\text { green innovative enterprise in } \\
\text { dynamic game model and } \\
\text { governmental policy making }\end{array}$ & $\begin{array}{c}\text { Science } \\
\text { Direct }\end{array}$ \\
\hline
\end{tabular}


Tabela 4. Continuação.

\begin{tabular}{|c|c|c|c|c|}
\hline Continente & Autoria & Ano & Título & Bases \\
\hline Asia & Mani et al. & 2014 & $\begin{array}{|lll|}\begin{array}{l}\text { Supplier selection using } \\
\text { sustainability: AHP } \\
\text { approach in India }\end{array} & & \text { based } \\
\end{array}$ & $\begin{array}{c}\text { Science } \\
\text { Direct }\end{array}$ \\
\hline Asia & Mary e Meenambal & 2016 & $\begin{array}{l}\text { Inventorisation of e-waste and } \\
\text { developing a policy: Bulk } \\
\text { consumer perspective }\end{array}$ & $\begin{array}{c}\text { Science } \\
\text { Direct }\end{array}$ \\
\hline Asia & Musa et al. & 2014 & $\begin{array}{l}\text { Enhancing the quality of life by } \\
\text { adopting sustainable modular } \\
\text { Industrialised Building System } \\
\text { (IBS) in the Malaysian } \\
\text { construction industry }\end{array}$ & $\begin{array}{c}\text { Science } \\
\text { Direct }\end{array}$ \\
\hline Asia & Nulkar & 2014 & $\begin{array}{l}\text { SMEs and environmental } \\
\text { performance: A framework for } \\
\text { green business strategies }\end{array}$ & $\begin{array}{c}\text { Science } \\
\text { Direct }\end{array}$ \\
\hline Asia & Qu et al. & 2015 & $\begin{array}{l}\text { Developing more insights on } \\
\text { sustainable consumption in China } \\
\text { based on Q methodology }\end{array}$ & Scopus \\
\hline Asia & Quoquab e Mohammad & 2016 & $\begin{array}{l}\text { Environment dominant logic: } \\
\text { Concerning for achieving the } \\
\text { sustainability marketing }\end{array}$ & $\begin{array}{c}\text { Science } \\
\text { Direct }\end{array}$ \\
\hline Asia & Rao & 2014 & 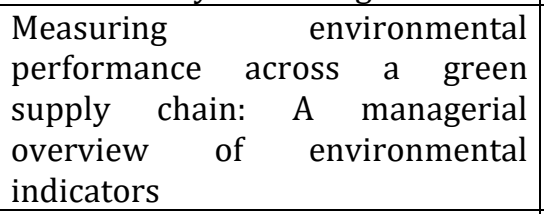 & Scopus \\
\hline Asia & Sahamir e Zakaria & 2014 & $\begin{array}{lrr}\text { Green } & \text { assessment } & \text { criteria for } \\
\text { public } & \text { hospital } & \text { building } \\
\text { development in Malaysia } & \\
\end{array}$ & $\begin{array}{c}\text { Science } \\
\text { Direct }\end{array}$ \\
\hline Asia & Sulistio e Rini & 2015 & $\begin{array}{l}\text { A structural literature review on } \\
\text { models and methods analysis of } \\
\text { green supply chain management }\end{array}$ & $\begin{array}{c}\text { Science } \\
\text { Direct }\end{array}$ \\
\hline Asia & Tseng et al. & 2014 & $\begin{array}{l}\text { Using TODIM to evaluate green } \\
\text { supply chain practices under } \\
\text { uncertainty }\end{array}$ & $\begin{array}{c}\text { Science } \\
\text { Direct }\end{array}$ \\
\hline Asia & Uslu et al. & 2015 & $\begin{array}{|lcr|}\text { Applicability } & \text { to } & \text { green } \\
\text { entrepreneurship } & \text { in } & \text { Turkey: A } \\
\text { situation analysis } & & \\
\end{array}$ & $\begin{array}{c}\text { Science } \\
\text { Direct }\end{array}$ \\
\hline Asia & Wang et al. & 2016 & \begin{tabular}{|lrr} 
Provincial & \multicolumn{2}{r}{ sustainability } \\
evaluation & based on the \\
standardized & national emergy \\
database & &
\end{tabular} & $\begin{array}{c}\text { Science } \\
\text { Direct }\end{array}$ \\
\hline Asia & $\mathrm{Xu}$ et al. & 2016 & $\begin{array}{l}\text { System establishment and method } \\
\text { application for quantitatively } \\
\text { evaluating the green degree of the } \\
\text { products in green public } \\
\text { procurement }\end{array}$ & Scopus \\
\hline Asia & Younis et al. & 2019 & $\begin{array}{l}\text { Green supply chain management } \\
\text { and corporate performance: } \\
\text { Developing a roadmap for future } \\
\text { research using a mixed method } \\
\text { approach }\end{array}$ & $\begin{array}{c}\text { Science } \\
\text { Direct }\end{array}$ \\
\hline
\end{tabular}


Tabela 4. Continuação.

\begin{tabular}{|c|c|c|c|c|}
\hline Continente & Autoria & Ano & Título & Bases \\
\hline Europa & Aldenius e Khan & 2017 & $\begin{array}{l}\text { Strategic use of green public } \\
\text { procurement in the bus sector: } \\
\text { Challenges and opportunities }\end{array}$ & $\begin{array}{l}\text { Science } \\
\text { Direct }\end{array}$ \\
\hline Europa & Ardente et al. & 2018 & $\begin{array}{l}\text { Accounting for the environmental } \\
\text { benefits of remanufactured } \\
\text { products: Method and application }\end{array}$ & $\begin{array}{l}\text { Science } \\
\text { Direct }\end{array}$ \\
\hline Europa & Bruce-Hyrkas et al. & 2018 & $\begin{array}{l}\text { Overview of whole building life- } \\
\text { cycle assessment for green } \\
\text { building certification and } \\
\text { ecodesign through industry } \\
\text { surveys and interviews }\end{array}$ & $\begin{array}{l}\text { Science } \\
\text { Direct }\end{array}$ \\
\hline Europa & Brzozowska et al. & 2015 & $\begin{array}{l}\text { Implementation of technical and } \\
\text { information systems in } \\
\text { environmental management }\end{array}$ & $\begin{array}{l}\text { Science } \\
\text { Direct }\end{array}$ \\
\hline Europa & Buchert et al. & 2015 & $\begin{array}{l}\text { Multi-criteria decision making as a } \\
\text { tool for sustainable product } \\
\text { development: Benefits and } \\
\text { obstacles }\end{array}$ & $\begin{array}{l}\text { Science } \\
\text { Direct }\end{array}$ \\
\hline Europa & Capasso et al. & 2019 & $\begin{array}{l}\text { Green growth: A synthesis of } \\
\text { scientific findings }\end{array}$ & $\begin{array}{c}\text { Science } \\
\text { Direct }\end{array}$ \\
\hline Europa & Cerutti et al. & 2018 & $\begin{array}{l}\text { Modelling, assessing, and ranking } \\
\text { public procurement options for a } \\
\text { climate-friendly catering service }\end{array}$ & Scopus \\
\hline Europa & Ciulli e Kolk & 2019 & $\begin{array}{l}\text { Incumbents and business model } \\
\text { innovation for the sharing } \\
\text { economy: Implications for } \\
\text { sustainability }\end{array}$ & $\begin{array}{l}\text { Science } \\
\text { Direct }\end{array}$ \\
\hline Europa & Cole et al. & 2019 & $\begin{array}{l}\begin{array}{l}\text { Assessing barriers to reuse of } \\
\text { electrical } \\
\text { equipment, a UK perspective }\end{array} \\
\text { equip }\end{array}$ & $\begin{array}{l}\text { Science } \\
\text { Direct }\end{array}$ \\
\hline Europa & Costa et al. & 2015 & $\begin{array}{l}\text { Sustainable product-service } \\
\text { systems for an office furniture } \\
\text { manufacturer: How insights from } \\
\text { a pilot study can inform pss design }\end{array}$ & $\begin{array}{l}\text { Science } \\
\text { Direct }\end{array}$ \\
\hline Europa & Crafoord et al. & 2018 & $\begin{array}{l}\text { The use of public procurement to } \\
\text { incentivize longer lifetime and } \\
\text { remanufacturing of computers }\end{array}$ & $\begin{array}{l}\text { Science } \\
\text { Direct }\end{array}$ \\
\hline Europa & Cristina & 2014 & $\begin{array}{l}\text { Saferational approach to a valid } \\
\text { sustainable development }\end{array}$ & $\begin{array}{c}\text { Science } \\
\text { Direct }\end{array}$ \\
\hline Europa & Duran et al. & 2015 & $\begin{array}{l}\text { The components of sustainable } \\
\text { development: A possible approach }\end{array}$ & $\begin{array}{c}\text { Science } \\
\text { Direct }\end{array}$ \\
\hline Europa & Dvoraková e Zborková & 2014 & $\begin{array}{l}\text { Integration of sustainable } \\
\text { development at enterprise level }\end{array}$ & $\begin{array}{l}\text { Science } \\
\text { Direct }\end{array}$ \\
\hline Europa & Edler e Yeow & 2016 & $\begin{array}{l}\text { Connecting demand and supply: } \\
\text { The role of intermediation in } \\
\text { public procurement of innovation }\end{array}$ & $\begin{array}{c}\text { Science } \\
\text { Direct }\end{array}$ \\
\hline Europa & Filippi e Sirombo & 2015 & $\begin{array}{l}\text { Green rating of existing school } \\
\text { facilities }\end{array}$ & $\begin{array}{c}\text { Science } \\
\text { Direct }\end{array}$ \\
\hline Europa & Foukaras e Toma & 2014 & $\begin{array}{l}\text { Buying and wasting sustainably: } \\
\text { Determinants of green behaviour } \\
\text { in Cyprus and Sweden }\end{array}$ & $\begin{array}{l}\text { Science } \\
\text { Direct }\end{array}$ \\
\hline
\end{tabular}


Tabela 4. Continuação.

\begin{tabular}{|c|c|c|c|c|}
\hline Continente & Autoria & Ano & Título & Bases \\
\hline Europa & Freidenfelds et al. & 2018 & $\begin{array}{l}\text { What does environmentally } \\
\text { sustainable higher education } \\
\text { institution mean? }\end{array}$ & $\begin{array}{c}\text { Science } \\
\text { Direct }\end{array}$ \\
\hline Europa & Geng et al. & 2017 & $\begin{array}{l}\text { The relationship between green } \\
\text { supply chain management and } \\
\text { performance: A meta-analysis of } \\
\text { empirical evidences in Asian } \\
\text { emerging economies }\end{array}$ & $\begin{array}{c}\text { Science } \\
\text { Direct }\end{array}$ \\
\hline Europa & Ghadimi e Heavey & 2014 & $\begin{array}{l}\text { Sustainable supplier selection in } \\
\text { medical device industry: Toward } \\
\text { sustainable manufacturing }\end{array}$ & $\begin{array}{c}\text { Science } \\
\text { Direct }\end{array}$ \\
\hline Europa & Ghisetti & 2017 & $\begin{array}{l}\text { Demand-pull and environmental } \\
\text { innovations: Estimating the effects } \\
\text { of innovative public procurement }\end{array}$ & $\begin{array}{c}\text { Science } \\
\text { Direct }\end{array}$ \\
\hline Europa & Hauschild & 2015 & $\begin{array}{l}\text { Better: But is it good enough? On } \\
\text { the need to consider both eco- } \\
\text { efficiency and eco-effectiveness to } \\
\text { gauge industrial sustainability }\end{array}$ & $\begin{array}{c}\text { Science } \\
\text { Direct }\end{array}$ \\
\hline Europa & Hayles & 2015 & $\begin{array}{l}\text { Environmentally } \\
\text { interior design: A snapshot of } \\
\text { current supply of and demand for } \\
\text { green, sustainable or fair trade } \\
\text { products for interior design } \\
\text { practice }\end{array}$ & $\begin{array}{c}\text { Science } \\
\text { Direct }\end{array}$ \\
\hline Europa & Hughes & 2017 & $\begin{array}{l}\text { The EU Circular Economy package: } \\
\text { Life cycle thinking to life cycle } \\
\text { law? }\end{array}$ & $\begin{array}{c}\text { Science } \\
\text { Direct }\end{array}$ \\
\hline Europa & Iacovidou et al. & 2017 & $\begin{array}{l}\text { Metrics for optimising the multi- } \\
\text { dimensional value of resources } \\
\text { recovered from waste in a circular } \\
\text { economy: A critical review }\end{array}$ & $\begin{array}{c}\text { Science } \\
\text { Direct }\end{array}$ \\
\hline Europa & Ivascu et al. & 2015 & $\begin{array}{l}\text { Modeling the green supply chain } \\
\text { in the context of sustainable } \\
\text { development }\end{array}$ & $\begin{array}{c}\text { Science } \\
\text { Direct }\end{array}$ \\
\hline Europa & Jedlinski & 2014 & $\begin{array}{l}\text { The position of green logistics in } \\
\text { sustainable development of a } \\
\text { smart green city }\end{array}$ & $\begin{array}{c}\text { Science } \\
\text { Direct }\end{array}$ \\
\hline Europa & Jonkutè & 2015 & $\begin{array}{l}\text { The consumers' approach to } \\
\text { sustainable consumption and } \\
\text { production: A case study in } \\
\text { Lithuania }\end{array}$ & Scopus \\
\hline Europa & Jørgensen e Remmen & 2018 & $\begin{array}{l}\text { A methodological approach to } \\
\text { development of circular economy } \\
\text { options in businesses }\end{array}$ & $\begin{array}{c}\text { Science } \\
\text { Direct }\end{array}$ \\
\hline Europa & Kalmykova et al. & 2018 & $\begin{array}{l}\text { Circular Economy: From review of } \\
\text { theories and practices to } \\
\text { development of implementation } \\
\text { tools }\end{array}$ & $\begin{array}{c}\text { Science } \\
\text { Direct }\end{array}$ \\
\hline Europa & Kern et al. & 2018 & $\begin{array}{l}\text { Sustainable software products: } \\
\text { Towards assessment criteria for } \\
\text { resource and energy efficiency }\end{array}$ & $\begin{array}{c}\text { Science } \\
\text { Direct }\end{array}$ \\
\hline
\end{tabular}


Tabela 4. Continuação.

\begin{tabular}{|c|c|c|c|c|}
\hline Continente & Autoria & Ano & Título & Bases \\
\hline Europa & Knight et al. & 2015 & $\begin{array}{l}\text { Supply market uncertainty: } \\
\text { Exploring } \\
\text { responses } \\
\text { transitions }\end{array}$ & $\begin{array}{c}\text { Science } \\
\text { Direct }\end{array}$ \\
\hline Europa & Konys & 2018 & $\begin{array}{l}\text { An ontology-based knowledge } \\
\text { modelling for a sustainability } \\
\text { assessment domain }\end{array}$ & Scopus \\
\hline Europa & Konys & 2019 & $\begin{array}{l}\text { Green supplier selection criteria: } \\
\text { From a literature review to a } \\
\text { comprehensive knowledge base }\end{array}$ & Scopus \\
\hline Europa & Kravchenko et al. & 2019 & $\begin{array}{l}\text { Implications of developing a tool } \\
\text { for sustainability screening of } \\
\text { circular economy initiatives }\end{array}$ & $\begin{array}{c}\text { Science } \\
\text { Direct }\end{array}$ \\
\hline Europa & Kuittinen & 2015 & $\begin{array}{l}\text { Setting the carbon footprint } \\
\text { criteria for public construction } \\
\text { projects }\end{array}$ & $\begin{array}{c}\text { Science } \\
\text { Direct }\end{array}$ \\
\hline Europa & Leendertse et al. & 2016 & $\begin{array}{l}\text { Sustainable market involvement in } \\
\text { transport } \\
\text { management }\end{array}$ & $\begin{array}{c}\text { Science } \\
\text { Direct }\end{array}$ \\
\hline Europa & Lintukangas et al. & 2019 & $\begin{array}{l}\text { The role of supply management } \\
\text { innovativeness and supplier } \\
\text { orientation in firms' sustainability } \\
\text { performance }\end{array}$ & $\begin{array}{c}\text { Science } \\
\text { Direct }\end{array}$ \\
\hline Europa & Luqmani et al. & 2017 & $\begin{array}{l}\text { Factors behind sustainable } \\
\text { business innovation: The case of a } \\
\text { global carpet manufacturing } \\
\text { company }\end{array}$ & $\begin{array}{c}\text { Science } \\
\text { Direct }\end{array}$ \\
\hline Europa & Milios & 2018 & $\begin{array}{l}\text { Advancing to a Circular Economy: } \\
\text { Three essential ingredients for a } \\
\text { comprehensive policy mix }\end{array}$ & Scopus \\
\hline Europa & Miska et al. & 2018 & $\begin{array}{l}\text { Culture's effects on corporate } \\
\text { sustainability practices: A multi- } \\
\text { domain and multi-level view }\end{array}$ & $\begin{array}{c}\text { Science } \\
\text { Direct }\end{array}$ \\
\hline Europa & Mityagin et al. & 2017 & $\begin{array}{l}\text { Methodology of estimation of } \\
\text { achieving regional goals of } \\
\text { sustainable development on the } \\
\text { basis of program and goal oriented } \\
\text { approach }\end{array}$ & $\begin{array}{c}\text { Science } \\
\text { Direct }\end{array}$ \\
\hline Europa & Moldavska e Welo & 2019 & $\begin{array}{l}\text { A Holistic approach to corporate } \\
\text { sustainability assessment: } \\
\text { Incorporating gustainable } \\
\text { development goals into } \\
\text { sustainable } \\
\text { performance evaluation }\end{array}$ & $\begin{array}{c}\text { Science } \\
\text { Direct }\end{array}$ \\
\hline Europa & Montalbán-Domingo et al. & 2019 & $\begin{array}{l}\text { Social sustainability in delivery } \\
\text { and procurement of public } \\
\text { construction contracts }\end{array}$ & Scopus \\
\hline Europa & Moschetti e Brattebo & 2016 & $\begin{array}{l}\text { Sustainable business models for } \\
\text { deep energy retrofitting of } \\
\text { buildings: State-of-the-art and } \\
\text { methodological approach }\end{array}$ & $\begin{array}{c}\text { Science } \\
\text { Direct }\end{array}$ \\
\hline
\end{tabular}


Tabela 4. Continuação.

\begin{tabular}{|c|c|c|c|c|}
\hline Continente & Autoria & Ano & Título & Bases \\
\hline Europa & Muller et al. & 2018 & $\begin{array}{l}\text { Life cycle rating: An approach to } \\
\text { support the decision-making } \\
\text { process of manufacturing systems }\end{array}$ & $\begin{array}{c}\text { Science } \\
\text { Direct }\end{array}$ \\
\hline Europa & Oviir & 2016 & $\begin{array}{l}\text { Life Cycle Assessment (LCA) in the } \\
\text { framework of the next generation } \\
\text { estonian building standard } \\
\text { building certification as a strategy } \\
\text { for enhancing sustainability }\end{array}$ & $\begin{array}{c}\text { Science } \\
\text { Direct }\end{array}$ \\
\hline Europa & Penz et al. & 2019 & $\begin{array}{l}\text { Explaining consumer choice of low } \\
\text { carbon footprint goods using the } \\
\text { behavioral spillover effect in } \\
\text { German-speaking countries }\end{array}$ & $\begin{array}{c}\text { Science } \\
\text { Direct }\end{array}$ \\
\hline Europa & Prieto-Sandoval et al. & 2018 & $\begin{array}{l}\text { Towards a consensus on the } \\
\text { circular economy }\end{array}$ & $\begin{array}{c}\text { Science } \\
\text { Direct }\end{array}$ \\
\hline Europa & Quak et al. & 2019 & $\begin{array}{l}\text { Public procurement as driver for } \\
\text { more sustainable urban freight } \\
\text { transport }\end{array}$ & $\begin{array}{c}\text { Science } \\
\text { Direct }\end{array}$ \\
\hline Europa & Riel et al. & 2015 & $\begin{array}{l}\text { An innovative approach to } \\
\text { teaching sustainable design and } \\
\text { management }\end{array}$ & $\begin{array}{c}\text { Science } \\
\text { Direct }\end{array}$ \\
\hline Europa & Ritzén e Sandstrom & 2017 & $\begin{array}{l}\text { Barriers to the Circular Economy: } \\
\text { Integration of perspectives and } \\
\text { domains }\end{array}$ & $\begin{array}{c}\text { Science } \\
\text { Direct }\end{array}$ \\
\hline Europa & Rogetzer et al. & 2019 & $\begin{array}{l}\text { Sustainable sourcing including } \\
\text { capacity reservation for recycled } \\
\text { materials: A newsvendor } \\
\text { framework with price and demand } \\
\text { correlations }\end{array}$ & $\begin{array}{c}\text { Science } \\
\text { Direct }\end{array}$ \\
\hline Europa & Russo et al. & 2019 & $\begin{array}{l}\text { From trash to treasure: The } \\
\text { impact of consumer perception of } \\
\text { bio-waste products in closed-loop } \\
\text { supply chains }\end{array}$ & $\begin{array}{c}\text { Science } \\
\text { Direct }\end{array}$ \\
\hline Europa & Santoyo-Castelazo e Adisa & 2014 & $\begin{array}{l}\text { Sustainability assessment of } \\
\text { energy systems: Integrating } \\
\text { environmental, economic and } \\
\text { social aspects }\end{array}$ & $\begin{array}{c}\text { Science } \\
\text { Direct }\end{array}$ \\
\hline Europa & Scherz et al. & 2018 & $\begin{array}{l}\text { Visualizing interdependencies } \\
\text { among sustainability criteria to } \\
\text { support multicriteria decision- } \\
\text { making processes in building } \\
\text { design }\end{array}$ & $\begin{array}{c}\text { Science } \\
\text { Direct }\end{array}$ \\
\hline Europa & Secher et al. & 2018 & $\begin{array}{l}\text { Construction product declarations } \\
\text { and sustainable development } \\
\text { goals for small and medium } \\
\text { construction enterprises }\end{array}$ & $\begin{array}{c}\text { Science } \\
\text { Direct }\end{array}$ \\
\hline Europa & Simion et al. & 2019 & $\begin{array}{l}\text { Green procurement in Romanian } \\
\text { construction projects. A cluster } \\
\text { analysis of the barriers and } \\
\text { enablers to green procurement in } \\
\text { construction projects from the } \\
\text { Bucharest-Ilfov Region of Romania }\end{array}$ & Scopus \\
\hline
\end{tabular}


Tabela 4. Continuação.

\begin{tabular}{|c|c|c|c|c|}
\hline Continente & Autoria & Ano & Título & Bases \\
\hline Europa & Sobotka e Sagan & 2016 & 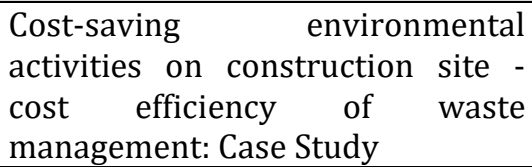 & $\begin{array}{c}\text { Science } \\
\text { Direct }\end{array}$ \\
\hline Europa & $\begin{array}{l}\text { Stasiak-Betlejewska e } \\
\text { Potkány }\end{array}$ & 2015 & $\begin{array}{l}\text { Construction costs analysis and its } \\
\text { importance to the economy }\end{array}$ & $\begin{array}{c}\text { Science } \\
\text { Direct }\end{array}$ \\
\hline Europa & Streimikiene & 2015 & $\begin{array}{l}\text { Environmental indicators for the } \\
\text { assessment of quality of life }\end{array}$ & $\begin{array}{c}\text { Science } \\
\text { Direct }\end{array}$ \\
\hline Europa & Thongplew et al. & 2017 & $\begin{array}{l}\text { Companies in search of the green } \\
\text { consumer: } \\
\text { consumption and production } \\
\text { strategies of companies and } \\
\text { intermediary organizations in } \\
\text { Thailand }\end{array}$ & $\begin{array}{l}\text { Science } \\
\text { Direct }\end{array}$ \\
\hline Europa & Uyarra et al. & 2014 & $\begin{array}{l}\text { Barriers to innovation through } \\
\text { public procurement: A supplier } \\
\text { perspective }\end{array}$ & $\begin{array}{l}\text { Science } \\
\text { Direct }\end{array}$ \\
\hline Europa & Watróbski & 2019 & $\begin{array}{lcc}\text { Ontology } & \text { supporting } & \text { green } \\
\text { supplier selection process } & \\
\end{array}$ & Scopus \\
\hline Europa & Witjes e Lozano & 2016 & $\begin{array}{l}\text { Towards a more Circular } \\
\text { Economy: Proposing a framework } \\
\text { linking sustainable public } \\
\text { procurement and sustainable } \\
\text { business models }\end{array}$ & $\begin{array}{l}\text { Science } \\
\text { Direct }\end{array}$ \\
\hline Europa & Zausková et al. & 2015 & $\begin{array}{l}\text { Marketing communication in eco- } \\
\text { innovation process }\end{array}$ & $\begin{array}{c}\text { Science } \\
\text { Direct }\end{array}$ \\
\hline Oceania & Harris e Divakarla & 2017 & $\begin{array}{l}\text { Supply chain risk to reward: } \\
\text { Responsible procurement and the } \\
\text { role of ecolabels }\end{array}$ & $\begin{array}{l}\text { Science } \\
\text { Direct }\end{array}$ \\
\hline Oceania & Khoshbakht et al. & 2017 & $\begin{array}{l}\text { Cost-benefit prediction of green } \\
\text { buildings: SWOT analysis of } \\
\text { research methods and recent } \\
\text { applications }\end{array}$ & $\begin{array}{l}\text { Science } \\
\text { Direct }\end{array}$ \\
\hline Oceania & Senaratne et al. & 2017 & $\begin{array}{l}\text { Recycled concrete in structural } \\
\text { applications for sustainable } \\
\text { construction practices in Australia }\end{array}$ & $\begin{array}{l}\text { Science } \\
\text { Direct }\end{array}$ \\
\hline
\end{tabular}

* Publicação de autores brasileiros.

Outro ponto importante a destacar, é que das sete publicações do continente americano, três são de autoria de brasileiros (Tabela 4).

É importante destacar que as 111 publicações selecionadas, de alguma forma atenderam direta ou indiretamente a pesquisa, por terem em seus trabalhos alguma informação que contribuiu com a pesquisa realizada. Porém, essas seis publicações atenderam diretamente e de forma clara e objetiva o tema compras públicas sustentáveis ou somente compras sustentáveis (Tabela 5). 
Tabela 5. Estudos selecionados que falam diretamente sobre compras sustentáveis.

\begin{tabular}{|c|c|c|c|c|c|}
\hline Autoria & Ano & Título & Bases & $\begin{array}{c}\text { Qualificação } \\
\text { Qualis }\end{array}$ & $\begin{array}{l}\text { Resumo do contexto que } \\
\text { direciona ao tema } \\
\text { compras sustentável }\end{array}$ \\
\hline $\begin{array}{l}\text { Foukaras e } \\
\text { Toma }\end{array}$ & 2014 & $\begin{array}{l}\text { Buying and wasting } \\
\text { sustainably. } \\
\text { Determinants of } \\
\text { green behaviour in } \\
\text { Cyprus and Sweden }\end{array}$ & $\begin{array}{l}\text { Procedia } \\
\text { Economics } \\
\text { and Finance }\end{array}$ & B2 & $\begin{array}{l}\text { Descreve sobre compra de } \\
\text { produtos com rótulo } \\
\text { ecológico e a separação } \\
\text { resíduos para reciclagem }\end{array}$ \\
\hline $\begin{array}{l}\text { Aldenius, e } \\
\text { Khan }\end{array}$ & 2017 & $\begin{array}{l}\text { Strategic use of } \\
\text { green public } \\
\text { procurement in the } \\
\text { bus sector: } \\
\text { Challenges and } \\
\text { opportunities }\end{array}$ & $\begin{array}{l}\text { Journal of } \\
\text { Cleaner } \\
\text { Production }\end{array}$ & $\mathrm{A} 1$ & $\begin{array}{l}\text { Descreve sobre desafios e } \\
\text { oportunidades na } \\
\text { realização das compras } \\
\text { públicas sustentáveis }\end{array}$ \\
\hline $\begin{array}{l}\text { Montalbán- } \\
\text { Domingo et } \\
\text { al. }\end{array}$ & 2019 & $\begin{array}{l}\text { Social Sustainability } \\
\text { in Delivery and } \\
\text { Procurement of } \\
\text { Public Construction } \\
\text { Contracts }\end{array}$ & ASCE Library & - & $\begin{array}{c}\text { Trata-se de uma análise } \\
\text { sobre a influência dos } \\
\text { métodos de e } \\
\text { procedimentos de compras } \\
\text { quanto à inserção de } \\
\text { critérios sociais nas } \\
\text { compras públicas } \\
\text { sustentáveis } \\
\end{array}$ \\
\hline $\begin{array}{l}\text { Crafoord et } \\
\text { al. }\end{array}$ & 2018 & $\begin{array}{l}\text { The use of public } \\
\text { procurement to } \\
\text { incentivize longer } \\
\text { lifetime and } \\
\text { remanufacturing of } \\
\text { computers }\end{array}$ & $\begin{array}{l}\text { Procedia } \\
\text { CIRP }\end{array}$ & B4 & $\begin{array}{l}\text { Descreve o número } \\
\text { crescente de inclusão de } \\
\text { critérios sustentáveis nas } \\
\text { compras públicas de } \\
\text { computadores, fatos } \\
\text { relevantes sobre seu ciclo } \\
\text { de vida, barreiras e a } \\
\text { abertura para novas } \\
\text { soluções inovadoras }\end{array}$ \\
\hline $\begin{array}{l}\text { Witjes } \\
\text { Lozano }\end{array}$ & 2016 & $\begin{array}{c}\text { Towards a more } \\
\text { Circular Economy: } \\
\text { Proposing a } \\
\text { framework linking } \\
\text { sustainable public } \\
\text { procurement and } \\
\text { sustainable } \\
\text { business models }\end{array}$ & $\begin{array}{c}\text { Resources, } \\
\text { Conservation } \\
\text { and Recycling }\end{array}$ & A1 & $\begin{array}{l}\text { Trata-se de uma proposta } \\
\text { de colaboração entre } \\
\text { compradores e } \\
\text { fornecedores, para buscar } \\
\text { reduções na utilização de } \\
\text { matéria-prima e na geração } \\
\text { de resíduos, promovendo o } \\
\text { desenvolvimento de } \\
\text { modelos de negócios mais } \\
\text { sustentáveis, contribuindo } \\
\text { melhor para tornar as } \\
\text { sociedades mais } \\
\text { sustentáveis. }\end{array}$ \\
\hline $\begin{array}{l}\text { Buniamin } \\
\text { et al. }\end{array}$ & 2015 & $\begin{array}{l}\text { Green Government } \\
\text { Procurement } \\
\text { Practices (GGP) in } \\
\text { Malaysian Public } \\
\text { Enterprise }\end{array}$ & $\begin{array}{l}\text { Procedia } \\
\text { Economics } \\
\text { and Finance }\end{array}$ & B2 & $\begin{array}{c}\text { O estudo explora as } \\
\text { percepções dos } \\
\text { profissionais da área } \\
\text { compras sobre as práticas } \\
\text { de compras públicas } \\
\text { sustentáveis, assim como, } \\
\text { examina as barreiras que } \\
\text { existem para a prática das } \\
\text { compras públicas } \\
\text { sustentáveis. } \\
\end{array}$ \\
\hline
\end{tabular}

Rev. Bras. Gest. Amb. Sustent., 2020, vol. 7, n. 17, p. 1391-1423. 


\section{Discussão}

Com a alteração do art. 3o, da Lei no 8.666/1993 (Brasil, 1993), pela Lei no 12.349/2010 (Brasil, 2010), que incluiu o desenvolvimento nacional sustentável como um dos princípios das licitações e a regulamentação desta alteração pelo Decreto no 7.746/2012 (Brasil, 2012), que estabelece critérios e práticas para a promoção do desenvolvimento nacional sustentável por toda a Administração Pública, fica instituído a obrigação da realização das compras públicas sustentáveis.

Além destas normas, recentemente foi publicado o Decreto no $10.024 / 2019$, que regulamenta a licitação na modalidade pregão eletrônico, onde também foi incluído como um dos seus princípios o desenvolvimento sustentável. Descrevendo como esse princípio se dará nas licitações, onde deverá ser observado, nas etapas do processo de contratação, as "dimensões econômica, social, ambiental e cultural, no mínimo, com base nos planos de gestão de logística sustentável dos órgãos e das entidades" (Brasil, 2019).

Diante desses fatores, os instrumentos convocatórios, deverão, dentro do possível, prever, tanto nas suas regras, quanto nas especificações dos objetos das contratações, práticas e critérios sustentáveis.

Portanto, diante dessa nova perspectiva, há a necessidade de discutir novas ações e estar disposto a mudanças culturais institucionais, porque apesar das alterações nas previsões legais, ainda há resistências quanto a esses novos paradigmas relacionados às compras públicas sustentáveis e barreiras, que precisam ser eliminadas. Diante disso, o presente artigo buscou oferecer contribuições relacionadas a experiências obtidas em outros estudos, que de alguma forma obtiveram respostas positivas, como uma forma de aperfeiçoar e aumentar às compras públicas sustentáveis no Brasil e assim fomentar a promoção do desenvolvimento nacional.

Os resultados do mapeamento sistemático de literatura têm uma abordagem de funil, começando com a apresentação de descobertas gerais sobre publicações que falam de desenvolvimento sustentável. Posteriormente, os estudos vão focando no tema de compras públicas sustentáveis.

Nos resultados obtidos, pode-se observar que existem poucos estudos que discutem especificamente sobre compras públicas sustentáveis, isso se torna um limitador para outros pesquisadores desenvolverem estudos com mais dados e até mesmo para estudarem outros temas relativos às compras públicas sustentáveis ou que são parte deste.

A ausência de estudos sobre o tema pode ser um empecilho para o aprimoramento e a melhoria das compras públicas sustentáveis na administração pública, não só do Brasil como de outros países. Pois essa ausência causa no desconhecimento de quem atua na área diretamente com ações fora do contexto da sustentabilidade. Estudos mais aprofundados sobre o tema podem permitir ações mais qualificadas e eficientes para que as compras públicas sustentáveis possam contribuir com a promoção do princípio do desenvolvimento sustentável no país.

Esta contribuição poderia vir de estudos que impulsionem inovações nas compras públicas sustentáveis, podendo ser até tecnológicas quanto à prática das atividades de compras com mais foco nas questões sustentáveis, como na construção de critérios mais concisos e focados para cada tipo de contratação, possibilitando a elaboração e um estudo melhor sobre indicadores sustentáveis que poderiam aperfeiçoar cada vez mais as compras públicas sustentáveis.

Cabe destacar, que é preciso também que as pessoas responsáveis pelas compras públicas sustentáveis entendam e conheçam sobre o que são as questões sustentáveis, como elas se dão e qual a importância de cada uma delas nesse contexto das compras públicas sustentáveis. Entender e conhecer melhor sobre a importância de se trabalhar o 
desenvolvimento sustentável numa determinada atividade, nesse caso, nas compras públicas sustentáveis.

Embora existam alguns estudos, até por parecerem não ser suficientes e, apesar das questões de sustentabilidade nas compras públicas estarem previstas desde 1999, quando se lançou a Agenda Ambiental da Administração Pública (A3P), ainda não há efetividade nas ações voltadas para sua prática. Poucos órgãos parecem aderir às compras públicas sustentáveis. Com mais estudos, mais pesquisas e discussões sobre o assunto, provavelmente, isso contribuiria para que se aumente o consumo de materiais e a contratação de serviços com critérios sustentáveis, licitações com regras de sustentabilidade, que promoveriam o desenvolvimento sustentável e ajudariam o país a preservar o planeta.

\section{Conclusão}

Neste artigo foram apresentados os resultados do mapeamento sistemático realizado. 0 processo de busca retornou 6.763 estudos, dos quais 306 inicialmente foram considerados relevantes e selecionados. Estes estudos, após a extração, diminuíram para 111 classificados como relevantes e de qualidade, que formaram uma base inicial para a pesquisa. O Science Direct foi a plataforma que mais contribuiu para o mapeamento, apresentando 5.430 artigos.

Os estudos selecionados apresentaram os seguintes desafios: avaliação da sustentabilidade, componentes do desenvolvimento sustentável, critérios sustentáveis, avaliação do impacto da avaliação do ciclo de vida, critérios de seleção de fornecedores, produção sustentável, consumo sustentável, comportamento diante das questões de sustentabilidade, cultura nas práticas corporativas de sustentabilidade, contabilização dos benefícios ambientais, sustentabilidade nos processos de obras, relacionamento comprador e fornecedor e barreiras em relação à aplicação dos assuntos relacionados a sustentabilidade.

Além desses, cabe destacar que a maioria dos estudos encontrados, apresentaram investigações sobre economia circular e gerenciamento de cadeia de suprimentos verdes, os quais apresentaram imaturidade em suas áreas de pesquisa e podem ser temas para futuros trabalhos. Apenas seis publicações selecionadas têm o tema com foco maior em compras sustentáveis, outras publicações o tema compras sustentáveis está implícito ou envolvem outras palavras chaves escolhidas para o estudo.

\section{Agradecimentos}

As autoras agradecem à Universidade Federal Rural do Rio de Janeiro (UFRRJ) e ao Instituto Federal de Educação, Ciência e Tecnologia do Rio de Janeiro (IFRJ) pelas facilidades concedidas para a realização desta pesquisa.

\section{Conflito de interesses}

As autoras declaram não haver conflito de interesses.

\section{Referências}

Aldenius, M.; Khan, J. Strategic use of green public procurement in the bus sector: Challenges and opportunities. Journal of Cleaner Production, v. 164, p. 250-257, 2017. https://doi.org/10.1016/j.jclepro.2017.06.196 
Ardente, F.; Peiró, L. T.; Matheiux, F.; Polverini, D. Accounting for the environmental benefits of remanufactured products: Method and application. Journal of Cleaner Production, v. 198, p. 1545-1558, 2018. https://doi.org/10.1016/j.jclepro.2018.07.012

Bae, H.S. The effect of environmental capabilities on environmental strategy and environmental performance of Korean exporters for green supply chain management. The Asian Journal of Shipping and Logistics, v. 33, p.167-176, 2017. https://doi.org/ 10.1016/j.ajsl.2017.09.006

Bansia, M.; Varkey, J. K.; Agrawal, S. Development of a reverse logistics performance measurement system for a battery manufacturer. Procedia Materials Science, v. 6, p. 1419-1427, 2014. https://doi.org/10.1016/j.mspro.2014.07.121

Biderman, R.; Macedo, L. S. V.; Monzoni, M.; Mazon, R. (Orgs.). Guia de compras públicas sustentáveis: uso do poder de compra do governo para a promoção do desenvolvimento sustentável. Rio de Janeiro: ICLEI, Editora FGV, 2008.

Bidin, Z. A.; Bohari, A. A. M.; Rais, S. L. A.; Saferi, M. M. Green related practices for construction procurement. IOP Conference Series: Earth and Environmental Science, v. 140, 012099, 2018. https://doi.org/10.1088/1755-1315/140/1/012099

Brasil. Decreto no 10.024, de 20 de setembro de 2019. Regulamenta a licitação, na modalidade pregão, na forma eletrônica, para a aquisição de bens e a contratação de serviços comuns, incluídos os serviços comuns de engenharia, e dispõe sobre o uso da dispensa eletrônica, no âmbito da administração pública federal. Disponível em: <http://www.in.gov.br/web/dou/-/decreto-n-10.024-de-20-de-setembro-de-2019$217537021>$. Acesso em: 26 abr. 2020.

Brasil. Decreto no 7.746, de 5 de junho de 2012. Regulamenta o art. 3o da Lei no 8.666, de 21 de junho de 1993, para estabelecer critérios e práticas para a promoção do desenvolvimento nacional sustentável nas contratações realizadas pela administração pública federal direta, autárquica e fundacional e pelas empresas estatais dependentes, e institui a Comissão Interministerial de Sustentabilidade na Administração Pública - CISAP. (Redação dada pelo Decreto $\mathrm{n}^{\mathrm{o}}$ 9.178, de 2017). Disponível em: <http://www.planalto.gov.br/ccivil_03/_Ato2011-2014/2012/Decreto/D7746.htm>. Acesso em: 06 jan. 2020.

Brasil. Lei no 12.349, de 15 de dezembro de 2010. Altera as Leis nos 8.666, de 21 de junho de 1993, 8.958, de 20 de dezembro de 1994, e 10.973, de 2 de dezembro de 2004; e revoga o $\S 1^{\mathrm{o}}$ do art. $2^{\circ}$ da Lei $\mathrm{n}^{\circ}$ 11.273, de 6 de fevereiro de 2006. Disponível em: <http://www.planalto.gov.br/ccivil_03/_Ato2007-2010/2010/Lei/L12349.htm>. Acesso em: 06 jan. 2020.

Brasil. Lei no 8.666, de 21 de junho de 1993. Regulamenta o art. 37, inciso XXI, da Constituição Federal, institui normas para licitações e contratos da Administração Pública e dá outras providências. Disponível em: <http://www.planalto.gov.br/ccivil_03/ leis/l8666cons.htm>. Acesso em: 06 jan. 2020.

Bruce-Hyrkas, T.; Pasanen, P.; Castro, R. Overview of whole building life-cycle assessment for green building certification and ecodesign through industry surveys and interviews. Procedia CIRP, v. 69, p. 178-183, 2018. https://doi.org/10.1016/j.procir.2017.11.127

Brzozowska, A.; Bubel, D.; Pabian, A. Implementation of technical and information systems in environmental management. Procedia - Social and Behavioral Sciences, v. 213, p. 992-999, 2015. https://doi.org/10.1016/j.procir.2014.07.110 
Buchert, T.; Neugebauer, S.; Schenker, S.; Lindow, K.; Stark, R. Multi-criteria Decision making as a tool for sustainable product development: Benefits and obstacles. Procedia CIRP, v. 26, p. 70-75, 2015. https://doi.org/10.1016/j.procir.2014.07.110

Buniamin, S.; Ahmad, N.; Rauf, F. H. A.; Johari, N. H.; Rashid, A. A. Green Government Procurement Practices (GGP) in Malaysian public enterprise. Procedia Economics and Finance, v. 35, p. 27-34, 2016. https://doi.org/10.1016/S2212-5671(16)00006-X

Capasso, M.; Hansen, T.; Heiberg, J.; Klitkou, A.; Steen, M. Green growth: A synthesis of scientific findings. Technological Forecasting and Social Change, v. 146, p. 390-402, 2019. https://doi.org/10.1016/j.techfore.2019.06.013

Cerutti, A. K.; Ardente, F.; Contu, S.; Donno, D.; Beccaro, G. L. Modelling, assessing, and ranking public procurement options for a climate-friendly catering service. International Journal of Life Cycle Assessment, v. 23, p. 95-115, 2018. https://doi.org/10.1007/ s11367-017-1306-y

Chin. T. A.; Tat, H. H.; Sulaiman, Z. Green supply chain management, environmental collaboration and sustainability performance. Procedia CIRP, v. 26, p. 695-699, 2015. https://doi.org/10.1016/j.procir.2014.07.035

Chun, S.-H.; Hwang, H. J.; Byun, Y.-H. Green supply chain management in the construction industry: Case of Korean construction companies. Procedia - Social and Behavioral Sciences, v. 186, p. 507-512, 2015a. https://doi.org/10.1016/j.sbspro.2015.04.192

Chun, S.-H.; Hwang, H. J.; Byun, Y.-H. Supply chain process and green business activities: Application to small and medium enterprises. Procedia - Social and Behavioral Sciences, v. 186, p. 862-867, 2015b. https://doi.org/10.1016/j.sbspro.2015.04.191

Ciulli, F.; Kolk, A. Incumbents and business model innovation for the sharing economy: Implications for sustainability. Journal of Cleaner Production, v. 214, p. 995-1010, 2019. https://doi.org/10.1016/j.jclepro.2018.12.295

Cole, C.; Gnanapragasam, A.; Cooper, T.; Singh, J. Assessing barriers to reuse of electrical and electronic equipment, a UK perspective. Resources, Conservation \& Recycling: $\mathbf{X}$, v. 1, p. 100004, 2019. https://doi.org/10.1016/j.rcrx.2019.100004

Costa, F.; Prendeville, S.; Beverley, K.; Teso, G.; Brooker, C. Sustainable product-service systems for an office furniture manufacturer: How insights from a pilot study can inform PSS design. Procedia CIRP, v. 30, p. 66-71, 2015. https://doi.org/10.1016/ j.procir.2015.02.109

Costa, B. B. F.; Motta, A. L. T. S. Key factors hindering sustainable procurement in the Brazilian Public sector: A Delphi study. International Journal of Sustainable Development and Planning, v. 14, p. 152-171, 2019. https://doi.org/10.2495/SDP-V14N2-152-171

Crafoord, K.; Dalhammar, C.; Milios, L. The use of public procurement to incentivize longer lifetime and remanufacturing of computers. Procedia CIRP, v. 73, p. 137-141, 2018. https://doi.org/10.1016/j.procir.2018.03.316

Cristina, M. D. Saferational approach to a valid sustainable development. Procedia Economics and Finance, v. 8, p. 497-504, 2014. https://doi.org/10.1016/S22125671(14)00119-1

Diyar, S.; Akparova, A.; Toktabayev, A.; Tyutunnikova, M. Green economy: Innovationbased development of Kazakhstan. Procedia - Social and Behavioral Sciences, v. 140, p. 695-699, 2014. https://doi.org/10.1016/j.sbspro.2014.04.497 
Duran, D. C.; Gogan, L. M.; Artene, A.; Duran, V. The components of sustainable development: A possible approach. Procedia Economics and Finance, v. 26, p. 806-811, 2015. https://doi.org/10.1016/S2212-5671(15)00849-7

Dvoraková, L.; Zborková, J. Integration of sustainable development at enterprise level. Procedia Engineering, v. 69, p.686-695, 2014. https://doi.org/10.1016/ j.proeng.2014.03.043

Edler, J.; Yeow, Y. Connecting demand and supply: The role of intermediation in public procurement of innovation. Research Policy, v. 45, p. 414-426, 2016. https://doi.org/ 10.1016/j.respol.2015.10.010

El Yamany, S.; Afifi, M.; Hassan, A. Applicability and implementation of U.S. Green Building Council Rating System (LEED) in Egypt (a longitudinal study for Egyptian LEED certified buildings). Procedia Environmental Sciences, v. 34, p. 594-604, 2016. https://doi.org/10.1016/j.proenv.2016.04.052

Eryigit, N.; Ozcure, G. Eco-innovation as modern era strategy of companies in developing countries: Comparison between Turkey and European Union. Procedia - Social and Behavioral Sciences, v. 195, p.1216-1225, 2015. https://doi.org/10.1016/ j.sbspro.2015.06.246

Falbo, R. A.; Souza, E. F.; Felizardo, K. R. Mapeamento sistemático. In: Felizardo, K.; Nakagawa, E.; Fabbri, S.; Ferrari, F. (Orgs.). Revisão sistemática da literatura em Engenharia de Software: teoria e prática. 1 ed. Rio de Janeiro: Elsevier, 2017. p. 79-98. Disponível em: <http://falbo.inf.ufes.br/files/MP/TP/Sobre_MS.pdf>. Acesso em: 06 jan. 2020.

Filippi, M.; Sirombo, E. Green rating of existing school facilities. Energy Procedia, v. 78, p. 3156-3161, 2015. https://doi.org/10.1016/j.egypro.2015.11.773

Foukaras, A.; Toma, L. Buying and wasting sustainably: Determinants of green behaviour in Cyprus and Sweden. Procedia Economics and Finance, v. 14, p. 200-229, 2014. https://doi.org/10.1016/S2212-5671(14)00705-9

Freidenfelds, D.; Kalnins, S. N.; Gusca, J. What does environmentally sustainable higher education institution mean? Energy Procedia, v. 147, p. 42-47, 2018. https://doi.org/ 10.1016/j.egypro.2018.07.031

Galankashi, M. R.; Chegeni, A.; Soleimanynanadegany, A.; Memari, A.; Anjomshoae. A.; Helmi, A. H.; Dargi, D. Prioritizing green supplier selection criteria using fuzzy analytical network process. Procedia CIRP, v. 26, p.689-694, 2015. https://doi.org/10.1016/ j.procir.2014.07.044

Geng, R.; Mansouri, A.; Aktas, E. The relationship between green supply chain management and performance: A meta-analysis of empirical evidences in Asian emerging economies. International Journal of Production Economics, v. 183, p. 245-258, 2017. https://doi.org/10.1016/j.ijpe.2016.10.008

Ghadimi, P.; Heavey, C. Sustainable supplier selection in medical device industry: Toward sustainable manufacturing. Procedia CIRP, v. 15, p.165-170, 2014. https://doi.org/ 10.1016/j.procir.2014.06.096

Ghisetti, C. Demand-pull and environmental innovations: Estimating the effects of innovative public procurement. Technological Forecasting and Social Change, v. 125, p. 178-187, 2017. https://doi.org/10.1016/j.techfore.2017.07.020

Gil, A. C. Métodos e técnicas de pesquisa social. 6. ed. São Paulo: Atlas, 2008. 
Harris, K.; Divakarla, S. Supply chain risk to reward: Responsible procurement and the role of ecolabels. Procedia Engineering, v. 180, p. 1603-1611, 2017. https://doi.org/10.1016/ j.proeng.2017.04.323

Hauschild, M. Better: But is it good enough? On the need to consider both eco-efficiency and eco-effectiveness to gauge industrial sustainability. Procedia CIRP, v. 29, p. 1-7, 2015. https://doi.org/10.1016/j.procir.2015.02.126

Hayles, C. Environmentally sustainable interior design: A snapshot of current supply of and demand for green, sustainable or fair trade products for interior design practice. International Journal of Sustainable Built Environment, v. 4, p. 100-108, 2015. https://doi.org/10.1016/j.ijsbe.2015.03.006

Hughes, R. The EU circular economy package: Life cycle thinking to life cycle law? Procedia CIRP, v. 61, p. 10-16, 2017. https://doi.org/10.1016/j.procir.2016.12.006

Iacovidou, E.; Velis, C. A.; Purnell, P.; Zwirner, O.; Brown, A.; Hahladakis, J.; MillwardHopkins, J.; Williams, P. T. Metrics for optimising the multi-dimensional value of resources recovered from waste in a circular economy: A critical review. Journal of Cleaner Production, v. 166, p. 910-938, 2017. https://doi.org/10.1016/j.jclepro.2017.07.100

ICLEI - Local Governments for Sustainability. Global lead city network on sustainable procurement. 2019. Disponível em: <https://glcn-on-sp.org/cities/>. Acesso em: 10 out. 2019.

Ingwersen, W.; Gausman, M.; Weisbrod, A.; Sengupta, D.; Lee, S.-J.; Bare, J.; Zanoli, E.; Bhander, G. S.; Ceja, M. Detailed life cycle assessment of Bounty® paper towel operations in the United States. Journal of Management in Engineering, v. 131, p. 509-522, 2016. https://doi.org/10.1016/j.jclepro.2016.04.149

Inti, S.; Sharma, M.; Tandon, V. An approach for performing life cycle impact assessment of pavements for evaluating alternative pavement designs. Procedia Engineering, v. 145, p. 964-971, 2016. https://doi.org/10.1016/j.proeng.2016.04.125

Irfan M.; Hassan, M.; Hassan, N. Unravelling the fuzzy effect of economic, social and environmental sustainability on the corporate reputation of public-sector organizations: A case study of Pakistan. Sustainability, v. 10, 2018. https://doi.org/10.3390/su10030769

Ivascu, L.; Mocan, M.; Draghici, A.; Turi, A.; Rus, S. Modeling the green supply chain in the context of sustainable development. Procedia Economics and Finance, v. 26, p. 702-708, 2015. https://doi.org/10.1016/S2212-5671(15)00819-9

Jayanti, R. K.; Gowda, M. V. R. Sustainability dilemmas in emerging economies. IIMB Management Review, v. 26, p. 130-142, 2014. https://doi.org/10.1016/ j.iimb.2014.03.004

Jayant, A.; Azhar, M. Analysis of the barriers for implementing Green Supply Chain Management (GSCM) practices: An Interpretive Structural Modeling (ISM) approach. Procedia Engineering, v. 97, p. 2157-2166, 2014. https://doi.org/10.1016/ j.proeng.2014.12.459

Jayant, A.; Gupta, P.; Garg, S. K. Simulation modelling and analysis of network design for closed-loop supply Chain: A case study of battery industry. Procedia Engineering, v. 97, p. 2213-2221, 2014. https://doi.org/10.1016/j.proeng.2014.12.465

Jedlinski, M. The position of green logistics in sustainable development of a smart green city. Procedia - Social and Behavioral Sciences, v. 151, p. 102-111, 2014. https://doi.org/10.1016/j.sbspro.2014.10.011 
Jonkutè, G. The consumers' approach to sustainable consumption and production: A case study in Lithuania. Environmental Research, Engineering and Management, v. 71, p. 28-46, 2015. https://doi.org/10.5755/j01.erem.71.4.13642

Jørgensen, M. S.; Remmen, A. A Methodological approach to development of circular economy options in businesses. Procedia CIRP, v. 69, p.816-821, 2018. https://doi.org/10.1016/j.procir.2017.12.002

Joshi, Y.; Rahman, Z. Factors affecting green purchase behaviour and future research directions. International Strategic Management Review, v. 3, p. 128-143, 2015. https://doi.org/10.1016/j.ism.2015.04.001

Kalmykova, Y.; Sadagopan, M.; Rosado, L. Circular economy: From review of theories and practices to development of implementation tools. Resources, Conservation and Recycling, v. 135, p. 190-201, 2018. https://doi.org/10.1016/j.resconrec.2017.10.034

Karimi, A.; Rahim, K. A. Classification of external stakeholders pressures in green supply chain management. Procedia Environmental Sciences, v. 30, p. 27-32, 2015. https://doi.org/10.1016/j.proenv.2015.10.005

Kern, E.; Hilty, L. M.; Guldner, A.; Maksimov, Y. V.; Filler, A.; Groger, J.; Naumann, S. Sustainable software products: Towards assessment criteria for resource and energy efficiency. Future Generation Computer Systems, v. 86, p.199-210, 2018. https://doi.org/10.1016/j.future.2018.02.044

Khoshbakht, M.; Gou, Z.; Dupre, K. Cost-benefit prediction of green buildings: SWOT analysis of research methods and recent applications. Procedia Engineering, v. 180, p. 167-178, 2017. https://doi.org/10.1016/j.proeng.2017.04.176

Kitchenham, B.; Brereton, O. P.; Budgen, D.; Turner, M.; Bailey, J.; Linkman, S. Systematic literature reviews in software engineering: A systematic literature review. Information and Software Technology, v. 51, n. 1, p. 7-15, 2009. https://doi.org/10.1016/ j.infsof.2008.09.009

Kitchenham, B.; Charters, S. Guidelines for performing systematic literature reviews in Software Engineering. Keele: Keele University, University of Durham, 2007. (Version 2.)

Kitchenham, B.; Pretorius, R.; Budgen, D.; Brereton, O. P.; Turner, M.; Niazi, M.; Linkman, S. Systematic literature reviews in software engineering: A tertiary study. Information and Software Technology, v. 52, n. 8, p. 792-805, 2010. https://doi.org/10.1016/ j.infsof.2010.03.006

Knight, L.; Pfeiffer, A.; Scott, J. Supply market uncertainty: Exploring consequences and responses within sustainability transitions. Journal of Purchasing and Supply Management, v. 21, p. 167-177, 2015. https://doi.org/10.1016/j.pursup.2015.04.002

Konys, A. An ontology-based knowledge modelling for a sustainability assessment domain. Sustainability, v. 11, 2018. https://doi.org/10.3390/su10020300

Konys, A. Green supplier selection criteria: From a literature review to a comprehensive knowledge base. Sustainability, v. 11, 2019. https://doi.org/10.3390/su11154208

Kravchenko, M.; McAloone, T. C.; Pigosso, D. C. A. Implications of developing a tool for sustainability screening of circular economy initiatives. Procedia CIRP, v. 80, p. 625-630, 2019. https://doi.org/10.1016/j.procir.2019.01.044 
Kuittinen, M. Setting the carbon footprint criteria for public construction projects. Procedia Economics and Finance, v. 21, p. 154-161, 2015. https://doi.org/10.1016/ S2212-5671(15)00162-8

Kumar, D.; Rahman, Z. Sustainability adoption through buyer supplier relationship across supply chain: A literature review and conceptual framework. International Strategic Management Review, v. 3, p. 110-127, 2015. https://doi.org/10.1016/j.ism.2015.04.002

Leendertse, W.; Arts, J.; Ridder, H. Sustainable market involvement in transport infrastructure management. Transportation Research Procedia, v. 14, p. 2936-2945, 2016. https://doi.org/10.1016/j.trpro.2016.05.414

Lintukangas K.; Kahkonen, A.-K.; Hallikas, J. The role of supply management innovativeness and supplier orientation in firms' sustainability performance. Journal of Purchasing and Supply Management, v. 25, p. 100558, 2019. https://doi.org/ 10.1016/j.pursup.2019.100558

Luqmani, A.; Leach, M.; Jesson, D. Factors behind sustainable business innovation: The case of a global carpet manufacturing company. Environmental Innovation and Societal Transitions, v. 24, p. 94-105, 2017. https://doi.org/10.1016/j.eist.2016.10.007

Ma, J.; Hou, A.; Tian, Y. Research on the complexity of green innovative enterprise in dynamic game model and governmental policy making. Chaos, Solitons \& Fractals: $\mathbf{X}$, v. 2, p. 100008, 2019. https://doi.org/10.1016/j.csfx.2019.100008

Mahdavinejad, M.; Zia, A.; Larki, A. N.; Ghanavati, S.; Elmi, N. Dilemma of green and pseudo green architecture based on LEED norms in case of developing countries. International Journal of Sustainable Built Environment, v. 3, p. 235-246, 2014. https://doi.org/ 10.1016/j.ijsbe.2014.06.003

Mani, V.; Agrawal, R.; Sharma, V. Supplier selection using social sustainability: AHP based approach in India. International Strategic Management Review, v. 2, p. 98-112, 2014. https://doi.org/10.1016/j.ism.2014.10.003

Mary, J. S.; Meenambal, T. Inventorisation of e-waste and developing a policy: Bulk consumer perspective. Procedia Environmental Sciences, v. 35, p. 643-655, 2016. https://doi.org/10.1016/j.proenv.2016.07.058

Milios, L. Advancing to a circular economy: Three essential ingredients for a comprehensive policy mix. Sustainability Science, v. 13, p.861-878, 2018. https://doi.org/10.1007/s11625-017-0502-9

Miska, C.; Szocs, I.; Schiffinger, M. Culture's effects on corporate sustainability practices: A multi-domain and multi-level view. Journal of World Business, v. 53, p. 263-279, 2018. https://doi.org/10.1016/j.jwb.2017.12.001

Mityagin, S. A.; Tikhonova, O. B.; Repkin A. I. Methodology of estimation of achieving regional goals of sustainable development on the basis of program and goal oriented approach. Procedia Computer Science, v. 108, p. 2038-2048, 2017. https://doi.org/ 10.1016/j.procs.2017.05.060

Moldavska, A.; Welo, T. A holistic approach to corporate sustainability assessment: Incorporating sustainable development goals into sustainable manufacturing performance evaluation. Journal of Manufacturing Systems, v. 50, p. 53-68, 2019. https://doi.org/ 10.1016/j.jmsy.2018.11.004

Montalbán-Domingo, L.; García-Segura, T.; Sanz, M. A.; Pellicer, E. Social sustainability in delivery and procurement of public construction contracts. Journal of Management in Engineering, v. 35, 2019. https://doi.org/10.1061/(ASCE)ME.1943-5479.0000674

Rev. Bras. Gest. Amb. Sustent., 2020, vol. 7, n. 17, p. 1391-1423. 
Moschetti, R.; Brattebo, H. Sustainable business models for deep energy retrofitting of buildings: State-of-the-art and methodological approach. Energy Procedia, v. 96, p. 435-445, 2016. https://doi.org/10.1016/j.egypro.2016.09.174

Muller, A.; Bornschlegl, M.; Mantwill, F. Life cycle rating: An approach to support the decision-making process of manufacturing systems. Procedia Manufacturing, v. 21, p. 305-312, 2018. https://doi.org/10.1016/j.promfg.2018.02.125

Musa, M. F.; Mohammad, F. M.; Mahbub, R.; Yusof, M. R. Enhancing the quality of life by adopting sustainable modular Industrialised Building System (IBS) in the Malaysian construction industry. Procedia - Social and Behavioral Sciences, v. 153, p. 79-89, 2014. https://doi.org/10.1016/j.sbspro.2014.10.043

Nulkar, G. SMEs and environmental performance: A framework for green business strategies. Procedia - Social and Behavioral Sciences, v. 133, p. 130-140, 2014. https://doi.org/10.1016/j.sbspro.2014.04.177

ONU - Organizações das Nações Unidas. Guia sobre desenvolvimento sustentável: 17 objetivos para transformar o nosso mundo. 2018. Disponível em: $<$ https://unric.org/pt/wp-content/uploads/sites/9/2019/01/SDG_brochure_PTweb.pdf>. Acesso em: 27 fev. 2020.

Oviir, A. Life Cycle Assessment (LCA) in the framework of the next generation Estonian building standard building certification as a strategy for enhancing sustainability. Energy Procedia, v. 96, p. 351-362, 2016. https://doi.org/10.1016/j.egypro.2016.09.159

Penz, E.; Hartl, B.; Hofmann, E. Explaining consumer choice of low carbon footprint goods using the behavioral spillover effect in German-speaking countries. Journal of Cleaner Production, v. 214, p. 429-439, 2019. https://doi.org/10.1016/j.jclepro.2018.12.270

Pereira, M.; Silveira, M. A necessidade de adaptação às regulações ambientais da Política Nacional de Resíduos Sólidos: do fabricante ao consumidor organizacional no setor de equipamentos eletromédicos. Innovation \& Management Review, v. 11, p. 88-109, 2015. https://doi.org/10.11606/rai.v11i4.100274

Petersen, K.; Feldt, R.; Mujtaba, S.; Mattsson, M. Systematic mapping studies in software engineering. Proceedings of the 12th International Conference on Evaluation and Assessment in Software Engineering, p. 68-77, 2008.

Petersen, K.; Vakkalanka, S.; Kuzniarz, L. Guidelines for conducting systematic mapping studies in software engineering: An update. Information and Software Technology, v. 64, p. 1-18, 2015. https://doi.org/10.1016/j.infsof.2015.03.007

Pimenta, H.C. D.; Ball, P. D. Analysis of environmental sustainability practices across upstream supply chain management. Procedia CIRP, v. 26, p.677-682, 2015. https://doi.org/10.1016/j.procir.2014.07.036

Prieto-Sandoval, V.; Jaca, C.; Ormazabal, M. Towards a consensus on the circular economy. Journal of Cleaner Production, v. 179, p.605-615, 2018. https://doi.org/10.1016/ j.jclepro.2017.12.224

Qu, Y.; Li, M.; Jia, H.; Guo, L. Developing more insights on sustainable consumption in China based on Q Methodology. Sustainability, v. 7, p. 14211-14229, 2015. https://doi.org/ $10.3390 /$ su71014211

Quak, H.; Nesterova, N.; Kok, R. Public procurement as driver for more sustainable urban freight transport. Transportation Research Procedia, v. 39, p. 428-439, 2019. https://doi.org/10.1016/j.trpro.2019.06.045 
Quoquab, F.; Mohammad, J. Environment dominant logic: Concerning for achieving the sustainability marketing. Procedia Economics and Finance, v. 37, p. 234-238, 2016. https://doi.org/10.1016/S2212-5671(16)30119-8

Rao, P. H. Measuring environmental performance across a green supply chain: A managerial overview of environmental indicators. Vikalpa, v. 39, p. 57-74, 2014. https://doi.org/10.1177/0256090920140104

Riel, A.; Lelah A.; Mandil, G.; Rio, M.; Tichkiewitch, S.; Zhang, F.; Zwolinski, P. An innovative approach to teaching sustainable design and management. Procedia CIRP, v. 36, p. 29-34, 2015. https://doi.org/10.1016/j.procir.2015.01.059

Ritzén, S.; Sandstrom, G. O. Barriers to the circular economy: Integration of perspectives and domains. Procedia CIRP, v. 64, p. 7-12, 2017. https://doi.org/10.1016/ j.procir.2017.03.005

Rogetzer, P.; Silbermayr, L.; Jammernegg, W. Sustainable sourcing including capacity reservation for recycled materials: A newsvendor framework with price and demand correlations. International Journal of Production Economics, v. 214, p. 206-219, 2019. https://doi.org/10.1016/j.ijpe.2019.03.014

Russo, I.; Confente, I.; Scarpi, D.; Hazen, B. T. From trash to treasure: The impact of consumer perception of bio-waste products in closed-loop supply chains. Journal of Cleaner Production, v. 218, p. 966-974, 2019. https://doi.org/10.1016/ j.jclepro.2019.02.044

Sahamir, S. R.; Zakaria, R. Green assessment criteria for public hospital building development in Malaysia. Procedia Environmental Sciences, v. 20, p. 106-115, 2014. https://doi.org/10.1016/j.proenv.2014.03.015

Santoyo-Castelazo, E.; Azapagic, A. Sustainability assessment of energy systems: Integrating environmental, economic and social aspects. Journal of Cleaner Production, v. 80, p. 119-138, 2014. https://doi.org/10.1016/j.jclepro.2014.05.061

Scherz, M.; Zunk, B. M.; Passer, A.; Kreiner, H. Visualizing interdependencies among sustainability criteria to support multicriteria decision-making processes in building design. Procedia CIRP, v. 69, p. 200-205, 2018. https://doi.org/10.1016/ j.procir.2017.11.115

Secher, A. Q.; Collin, C.; Linnet, A. Construction product declarations and sustainable development goals for small and medium construction enterprises. Procedia CIRP, v. 69, p. 54-58, 2018. https://doi.org/10.1016/j.procir.2017.12.011

Senaratne, S.; Lambrousis, G.; Mirza, O.; Tam, V. W. Y.; Kang, W.-H. Recycled concrete in structural applications for sustainable construction practices in Australia. Procedia Engineering, v. 180, p. 751-758, 2017. https://doi.org/10.1016/j.proeng.2017.04.235

Simion, C.-P.; Nicolescu, C.; Vrincut, M. Green procurement in Romanian construction projects. A cluster analysis of the barriers and enablers to green procurement in construction projects from the Bucharest-Ilfov Region of Romania. Sustainability, v. 11, n. 6231, 2019. https://doi.org/10.3390/su11226231

Skerlos, S. J. Promoting effectiveness in sustainable design. Procedia CIRP, v. 29, p. 13-18, 2015. https://doi.org/10.1016/j.procir.2015.02.080

Sobotka, A.; Sagan, J. Cost-saving environmental activities on construction site - cost efficiency of waste management: Case study. Procedia Engineering, v. 161, p. 388-393, 2016. https://doi.org/10.1016/j.proeng.2016.08.579 
Stasiak-Betlejewska, R.; Potkány, M. Construction costs analysis and its importance to the economy. Procedia Economics and Finance, v. 34, p. 35-42, 2015. https://doi.org/ 10.1016/S2212-5671(15)01598-1

Streimikiene, D. Environmental indicators for the assessment of quality of life. Intellectual Economics, v. 9, p.67-69, 2015. https://doi.org/10.1016/ j.intele.2015.10.001

Sulistio, J.; Rini, T. A. A structural literature review on models and methods analysis of green supply chain management. Procedia Manufacturing, v. 4, p. 291-299, 2015. https://doi.org/10.1016/j.promfg.2015.11.043

Thongplew, N.; Spaargaren, G.; Kris van Koppen, C. S. A. Companies in search of the green consumer: Sustainable consumption and production strategies of companies and intermediary organizations in Thailand. NJAS - Wageningen Journal of Life Sciences, v. 83, p. 12-21, 2017. https://doi.org/10.1016/j.njas.2017.10.004

Tseng, M.-L.; Lin, Y.-H.; Tan, K.; Chen, R.-H.; Chenl, Y.-H. Using TODIM to evaluate green supply chain practices under uncertainty. Applied Mathematical Modelling, v. 38, p. 2983-2995, 2014. https://doi.org/10.1016/j.apm.2013.11.018

Uslu, Y. D.; Hancioglu, Y.; Demir, E. Applicability to green entrepreneurship in Turkey: A situation analysis. Procedia - Social and Behavioral Sciences, v. 195, p. 1238-1245, 2015. https://doi.org/10.1016/j.sbspro.2015.06.266

Uyarra, E.; Edler, J.; Garcia-Estevez, J.; Georghiou, L.; Yeow, J. Barriers to innovation through public procurement: A supplier perspective. Technovation, v. 34, p. 631-645, 2014. https://doi.org/10.1016/j.technovation.2014.04.003

Vargas, C.; Mantilla, M. Organizational antecedents and capabilities for sustainable supply chain management in developing economies: The case of Colombian focal firms. Cuadernos de Administracion, v. 29, n. 3, p. 101-143, 2016. https://doi.org/10.11144/ Javeriana.cao29-53.oacs

Wang, C.; Liu, G.; Ren, S.; Liu, X.; Hao, Y. Provincial sustainability evaluation based on the standardized national emergy database. Energy Procedia, v. 88, p. 153-159, 2016. https://doi.org/10.1016/j.egypro.2016.06.040

Watróbski, J. Ontology supporting green supplier selection process. Procedia Computer Science, v. 159, p. 1602-1613, 2019. https://doi.org/10.1016/j.procs.2019.09.331

Witjes, S.; Lozano, R. Towards a more Circular Economy: Proposing a framework linking sustainable public procurement and sustainable business models. Resources, Conservation and Recycling, v. 112, p. 37-44, 2016. https://doi.org/10.1016/ j.resconrec.2016.04.015

$\mathrm{Xu}$, S.; Chu, C.; Ju, M.; Shao, C. System establishment and method application for quantitatively evaluating the green degree of the products in green public procurement. Sustainability, v. 8, n. 9, 2016. https://doi.org/10.3390/su8090941

Yadavalli, V. S.; Darbari, J. D.; Bhayana, N.; Jha, P. C.; Agarwal, V. An integrated optimization model for selection of sustainable suppliers based on customers' expectations. Operations Research Perspectives, v. 6, 100113, 2019. https://doi.org/10.1016/j.orp.2019.100113

Younis, H.; Sundarakani, B.; O’Mahony, B. Green supply chain management and corporate performance: Developing a roadmap for future research using a mixed method approach. IIMB Management Review, 2019. https://doi.org/10.1016/j.iimb.2019.10.011 
Zausková, A.; Bezáková, Z.; Grib, L. Marketing communication in eco-innovation process. Procedia Economics and Finance, v. 34, p.670-675, 2015. https://doi.org/10.1016/ S2212-5671(15)01684-6 